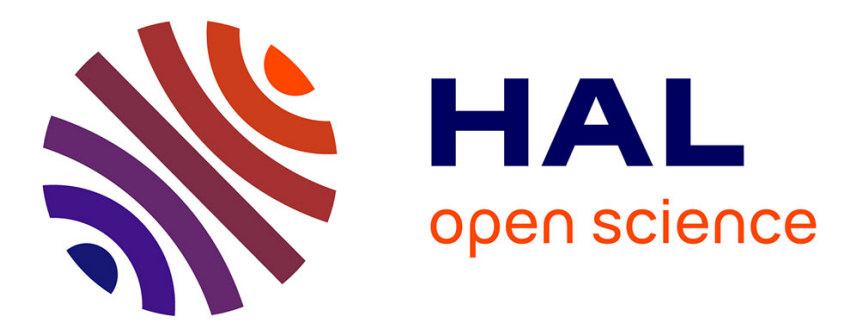

\title{
Flexural waves focusing through shunted piezoelectric patches
}

Kaijun Yi, Manuel Collet, Mohamed Ichchou, Lin Li

\section{To cite this version:}

Kaijun Yi, Manuel Collet, Mohamed Ichchou, Lin Li. Flexural waves focusing through shunted piezoelectric patches. Smart Materials and Structures, 2016, 25 (7), pp.075007. 10.1088/09641726/25/7/075007. hal-03316298

\section{HAL Id: hal-03316298 \\ https://hal.science/hal-03316298}

Submitted on 9 Aug 2021

HAL is a multi-disciplinary open access archive for the deposit and dissemination of scientific research documents, whether they are published or not. The documents may come from teaching and research institutions in France or abroad, or from public or private research centers.
L'archive ouverte pluridisciplinaire HAL, est destinée au dépôt et à la diffusion de documents scientifiques de niveau recherche, publiés ou non, émanant des établissements d'enseignement et de recherche français ou étrangers, des laboratoires publics ou privés. 


\title{
Flexural waves focusing through shunted piezoelectric patches
}

\author{
$\mathbf{K} \mathbf{Y i}^{1}, \mathbf{M}$ Collet ${ }^{1}, \mathbf{M}$ Ichchou $^{1}$ and $\mathbf{L} \mathbf{L i}^{2,3}$ \\ ${ }^{1}$ LTDS UMR5513 Ecole Centrale de Lyon, 36 Avenue Guy de Collongue, 69130 Ecully, France \\ ${ }^{2}$ School of Energy and Power Engineering, Beihang University, Beijing 100191, People's Republic of China \\ ${ }^{3}$ Co-Innovation Center for Advanced Aero-Engine, Beijing, People's Republic of China
}

E-mail: manuel.collet@ec-lyon.fr

\begin{abstract}
In this paper, we designed and analyzed a piezo-lens to focus flexural waves in thin plates. The piezo-lens is comprised of a host plate and piezoelectric arrays bonded on the surfaces of the plate. The piezoelectric patches are shunted with negative capacitance circuits. The effective refractive indexes inside the piezo-lens are designed to fit a hyperbolic secant distribution by tuning the negative capacitance values. A homogenized model of a piezo-mechanical system is adopted in the designing process of the piezo-lens. The wave focusing effect is studied by the finite element method. Numerical results show that the piezo-lens can focus flexural waves by bending their trajectories, and is effective in a large frequency band. The piezo-lens has the ability to focus flexural waves at different locations by tuning the shunting negative capacitance values. The piezo-lens is shown to be effective for flexural waves generated by different types of sources.
\end{abstract}

Keywords: wave focusing, piezoelectric patch, negative capacitance, homogenization, finite element modeling, power flow

\section{Introduction}

Wave focusing is a method which promises to be useful in applications such as structural health monitoring (SHM) [1], energy harvesting [2], etc. It is reported that acoustic waves $[3,4]$ and elastic waves [5, 6] can be focused by a flat slab composed of phononic crystals (PCs) at a certain frequency band. The main fault of this kind of focusing is that the effective frequency band is quite narrow. In order to obtain more broadband wave focusing, methods based on the gradient index (GRIN) were proposed. GRIN is often used to describe an inhomogeneous medium, in which the refractive index is dependent on spatial coordinates. In a GRIN medium, wave rays follow curved trajectories. Therefore, by an appropriate choice of the refractive index profile, a GRIN medium can focus waves. Generally, a GRIN medium that can focus waves is termed a GRIN lens in the literature
[1, 2, 7]. GRIN lenses are well known in optics [7], and in recent years, they have been introduced to focus acoustic waves and the lowest order Lamb waves in plate-like structures. No matter what kind of wave they are used for, the main challenge for the GRIN lens is the realization of the desired refractive index profile.

In the literature, for acoustic waves and elastic waves, GRIN lenses were realized by using PCs, metamaterials or geometry design. PCs are composite with a periodically arranged unit lattice. They behave at a low frequency band as homogenous materials with effective parameters mainly dependent on the unit lattice. Therefore, by locally changing the parameters of the unit lattice according to specific functions, artificial GRIN lenses can be obtained. Following this approach, GRIN lenses were designed for acoustic waves [8$10]$ and guided waves in a plate [2, 11, 12]. Metamaterials exhibit unusual effective dynamic properties and they show 
promise as alternative candidates to achieve GRIN lenses. This has been demonstrated, for instance, in the work of Yan et al [1]. In their work, the metamaterial is obtained by bonding a local resonator on a host plate's surface. The effective mass density of this metamaterial can be tuned by designing the local resonator. According to this, a GRIN lens was realized by periodically arranging the local resonators on the plate surface and tuning them to fit a required refractive index variation pattern. Geometry design is the third typical way to realize GRIN lenses. At a low frequency band, where the Kirchhoff-love plate theory holds, the wave numbers of A0 (the flexural wave) and S0 (the longitudinal wave) Lamb waves in a thin isotropic plate are dependent on the plate thickness. Based on this, Climente et al [13] developed two kinds of GRIN lenses in a plate by modifying the local thickness.

However, the existing GRIN lenses realized by PCs, metamaterials or geometry design face several challenges in practice. For example, the GRIN lenses composed of PCs or obtained by geometry design need to modify the original host structure; this may result in new problems. The GRIN lens made by local resonant metamaterials is only effective in a narrow frequency band. In order to obtain more advanced and practical GRIN lenses, a promising way is to use piezoelectric materials.

In the past few decades, piezoelectric materials were proposed for many applications. For example, piezoelectric materials were widely used in vibration suppression. Active and passive techniques were developed in this application [14]. In one kind of passive technique, piezoelectric patches are directly connected with passive external circuits. Theoretical analysis of such passive techniques were first carried out by Hagood and VonFlotow [15]. In their works, the resistive $(\mathrm{R})$ shunt and the resistive-inductive $(\mathrm{RL})$ shunt were involved. Accoding to their interpretation, the piezoelectric patch shunted through the $\mathrm{R}$ circuit has a similar effect to viscous damping; the piezoelectric patch with RL shunt acts like a vibration absorber at the resonance frequency of the circuit. Since then, more complex passive techniques of this kind were proposed to have better effectiveness. For example, multi-mode techniques [16, 17], negative capacitance circuits $[18,19]$ and distributed shunted piezoelectric patches [20]. In another kind of passive technique, the piezoelectric patches are periodically distributed on the controlled structures and are interconnected with an electric network. The key idea is to design the electric network, which is coupled with the elastic structure through the piezoelectric patches, to obtain wide band electromechanical energy exchange. For example, in the work of Alessandroni et al [21], they periodically distributed piezoelectric patches on the surface of a plate, and then designed an electric network to interconnect them. The designed electric network is mathematically analogous to a flexural plate. Multi-mode vibration suppression is obtained by tuning the parameters in the electric network to induce internal resonances between electric modes and structural modes.

Piezolectric materials were also adopted to control wave propagation and energy flow. For the wave propagation control, Thorp et al [22] periodically placed shunted piezoelectric patches along rods to control the longitudinal wave propagation in them. They found that at certain frequency ranges (namely stop bands) no propagative longitudinal waves exist and these stop bands can be extended or relocated by tuning the RL shunts. This concept was later extended to flexural beams [23] and plates [20, 24]. Piezoelectric patches shunted with negative capacitance circuits were also integrated into lattices of initial periodic structures to control band gaps [25] or directivities [26]. For the energy flow control, in [27], piezoelectric patches were periodically distributed on a host beam to form a periodic piezo-composite structure, and this functional beam was connected with a passive beam with identical parameters to the host beam. It was found that at the interface of these two different beams, by utilizing and optimizing negative capacitances as well as resistances, the total reflection or total absorption of certain types of waves incident from the passive beam can be achieved. In another work [28], an interface composed of a host plate and periodically distributed piezoelectric patches shunted with negative capacitances and resistances was arranged between two passive plates to diminish energy translated from one passive domain to another one by optimizing the shunting impedances. Its effectiveness was verified by experiments in Tateo et al's works [29, 30]. Another example of energy flow control can be found in Fan et al's work [31].

The prospect of piezoelectric materials in building GRIN lenses comes from the fact that their effective parameters can be controled by the shunting impedance. It was theoretically demonstrated by Hagood and Von Flotow [15] that when the electrodes of the piezoelectric patches are directly connected with an external electrical network, the effective material parameters of these shunted piezoelectric patches can be modified by tuning the shunting impedance. In particular, later research shows that when piezoelectric patches are shunted with negative capacitance circuits [29], their effective material parameters are independent of the frequency and can be modified to a great extent [32].

In this work, efforts are made to focus flexural waves in a thin plate by using shunted piezoelectric patches. Piezoelectric patches are periodically bonded on the upper and lower surfaces of the host plate in a collocated fashion to form a flat GRIN piezo-lens. Negative capacitance circuits are used to shunt the piezoelectric patches. An analytical relation between the negative capacitance value and the effective refractive index of the piezo-mechanical system is established by homogenizing the piezo-mechanical system. The piezolens is designed based on this relation. The focusing effects are studied by using the finite element method. In the numerical study, the focusing effect of the piezo-lens is verified and the adaptive ability is demonstrated. The performances of the piezo-lens at different frequencies are then analyzed. After that, the performances of the piezo-lens for flexural waves generated by different types of sources at different frequencies are estimated. A double piezo-lens configuration is proposed to focus waves excited by near field point forces. Discussions on the potential applications of the piezo-lens are also made based on the numerical results in the conclusion. 


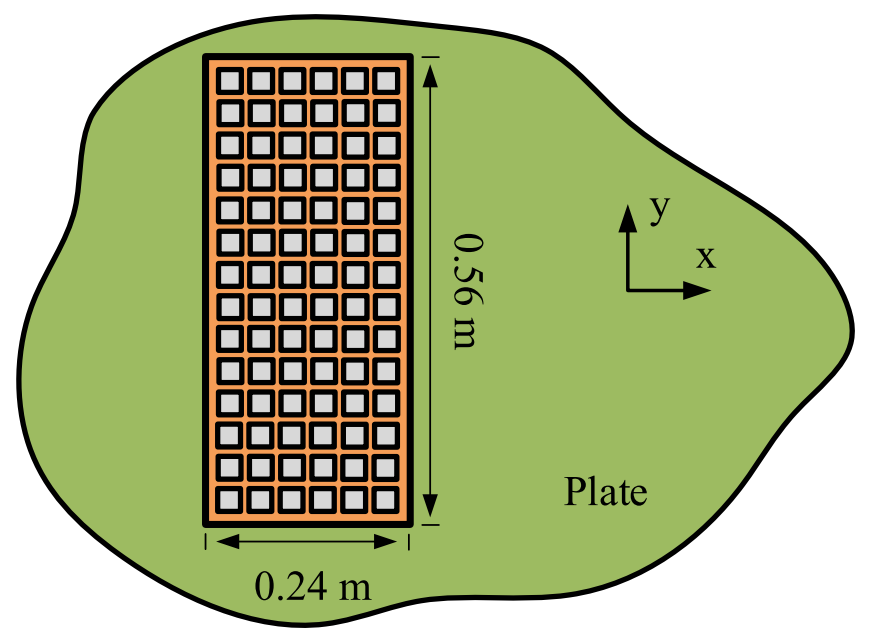

Figure 1. Piezo-lens on plate.

The remainder of this paper is organized into four sections. Section 2 introduces the piezo-lens model and describes the design process of the piezo-lens. Section 3 introduces the numerical model of the piezo-mechanical system and the energy analyses used in analyzing the numerical results. Section 4 presents the numerical results. Section 5 summarizes the remarkable conclusions and gives corresponding discussions.

\section{Piezo-lens model and design}

The piezo-lens in this study is comprised of an aluminum host plate and two 14-by-6 arrays of piezoelectric patches (PZT26) bonded respectively at the upper and lower surfaces of the host plate as depicted in figure 1. The host plate lies in the $x-$ $y$ plane and occupies the spatial region $-h_{b} / 2 \leqslant z \leqslant h_{b} / 2$. The piezoelectric patches are shunted with negative capacitance circuits. A detailed shunted piezoelectric lattice in the piezo-lens zone is shown in figure 2.

As proposed by [8], a flat GRIN lens to focus flexural waves in a thin plate can be obtained if the refractive index for the flexural wave inside the lens zone fulfills a hyperbolic secant function:

$$
n(y)=n_{0} \cdot \operatorname{sech}[\alpha(y-\beta)]
$$

in which, $n_{0}$ represents the refractive index of the background plate, $\alpha$ is the gradient coefficient and $\beta$ represents the $y$ coordinate of the symmetry axis of the refractive index profile. Waves incident into the lens from the $x$ direction will be focused at a focal point on the $y=\beta$ line, with a focal length represented as $f=\pi / 2 \alpha$.

In the considered piezo-lens, the above variation of the refractive index is approximately realized in a piecewise form by designing the shunting negative capacitance values. According to equation (1), the refractive index only varies in the $y$ direction. Thus, in a piezo-lens, the shunting negative capacitance values are equal in the same row (the $x$ direction) but will be different in the same column (the $y$ direction). To determine the required shunting negative capacitance value in

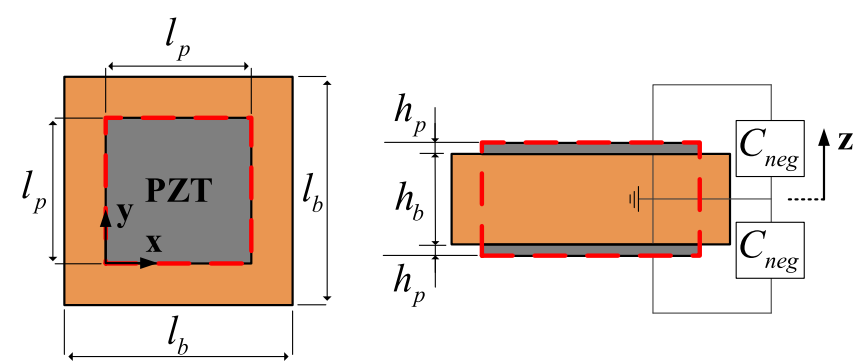

Figure 2. Top and side view of one unit shunted piezoelectric lattice.

each row, firstly, a homogenized model of the shunted piezoelectric lattice depicted in figure 2 is developed.

The homogenized model is obtained in three steps [33]. The first step is to represent the effective material parameters of the shunted piezoelectric patches as functions of the negative capacitance. Assume directions 1, 2 and 3 correspond to the $x, y, z$ axis respectively, and the electrode surfaces of the piezoelectric patches are perpendicular to direction 3. The thickness of the piezoelectric patches are much smaller than the host plate's thickness, therefore it is reasonable to assume that all piezoelectric patches are under plane stress condition and they are isotropic in the $x-y$ plane. According to Hagood and Von Flotow [15], the effective Young's modulus and the effective Poisson's ratio of these piezoelectric patches shunted with negative capacitances are:

$$
\begin{aligned}
& E_{p}=E_{p}^{s h} \frac{C_{n e g}+C_{p}^{T}}{C_{n e g}+C_{p}^{T}\left(1-k_{31}^{2}\right)} \\
& \mu_{p}=\mu_{p}^{s h} \frac{C_{n e g}+C_{p}^{T}\left(1+k_{31}^{2} / \mu_{p}^{s h}\right)}{C_{n e g}+C_{p}^{T}\left(1-k_{31}^{2}\right)} .
\end{aligned}
$$

Here, $E_{p}^{s h}$ and $\mu_{p}^{s h}$ are the Young's modulus and the Poisson's ratio of the short-circuit piezoelectric material, respectively; $k_{31}$ is the coupling factor; $C_{p}^{T}$ is the intrinsic capacitance value of the piezoelectric patch under constant stress; $C_{n e g}$ is the applied negative capacitance value.

The second step is to determine the effective parameters of the shunted piezoelectric sandwich structure highlighted by the dashed lines in figure 2. According to the classical laminated plate theory [34] as well as the Kirchhoff-Love plate theory, the effective area density, the effective flexural rigidity for the piezoelectric sandwich structure can be expressed as:

$$
\begin{aligned}
& \rho_{A}=\rho_{b} h_{b}+2 \rho_{p} h_{p} \\
& D_{A}=D_{b}+\frac{2 E_{p}}{3\left(1-\mu_{p}^{2}\right)}\left[\left(\frac{h_{b}}{2}+h_{p}\right)^{3}-\left(\frac{h_{b}}{2}\right)^{3}\right] .
\end{aligned}
$$

Here, $D_{b}=\frac{E_{b} h_{b}^{3}}{12\left(1-\mu_{b}^{2}\right)}$ is the flexural rigidity of the host plate and $E_{b}, \mu_{b}$ denote the Young's modulus and the Poisson's ratio of the host plate respectively; $\rho_{b}$ and $\rho_{p}$ are the densities of the host plate and the piezoelectric patches respectively.

The last step is to derive the effective parameters for the entire shunted piezoelectric lattice. With equations (2) and (3), 


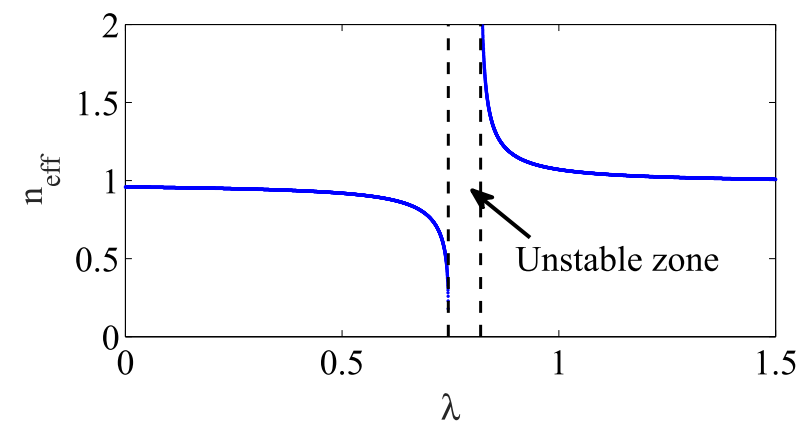

Figure 3. Variation of effective refractive index with non-dimensional applied negative capacitance value.

the effective area density and the effective flexural rigidity of the entire unit lattice can be obtained as [33]:

$$
\begin{aligned}
\rho_{\text {eff }} & =\chi \rho_{A}+(1-\chi) \rho_{b} h_{b} \\
D_{\text {eff }} & =\frac{D_{A} D_{b}}{\chi D_{A}+(1-\chi) D_{b}} .
\end{aligned}
$$

Here, $\chi=\left(l_{p} / l_{b}\right)^{2}$ is the ratio of the surface area covered by a piezoelectric patch to the surface area of a unit lattice.

After developing the homogenized model for the shunted piezoelectric lattice, the effective refractive index for flexural waves incident from the background plate into the shunted piezoelectric lattice is obtained. It is expressed as the ratio of the group velocity in the background plate to the effective group velocity in the lattice:

$$
n_{\text {eff }}=\frac{c_{b}^{g}}{c_{e f f}^{g}}
$$

For the homogeneous and isotropic plate, the group velocity of the flexural wave is double the phase velocity and is independent of the direction. Hence, equation (5) can be further written as:

$$
n_{e f f}=\left(\frac{\rho_{e f f} D_{b}}{\rho_{b} h_{b} D_{e f f}}\right)^{1 / 4} .
$$

Figure 3 illustrates the variation of the effective refractive index with the non-dimensional applied negative capacitance value $\lambda=-C_{n e g} / C_{p}^{T}$. It is observed that the effective refractive index can be increased or decreased to a great extent by varying the negative capacitance value in the stable zone.

With the relationship between the negative capacitance value and the effective refractive index of the shunted piezoelectric lattice shown in equation (6) or in figure 3 , the required negative capacitance value for each row in the piezolens can be determined.

As a summary of this section, the piezo-lens is designed in three steps. In the first step, we choose the parameters $\alpha$ and $\beta$ in the refractive index profile in equation (1) to design the location of the focal point. In the second step, the required refractive index for each row in the lens zone is obtained by substituting the central $y$ coordinate of the row into equation (1). In the last step, the required refractive index for

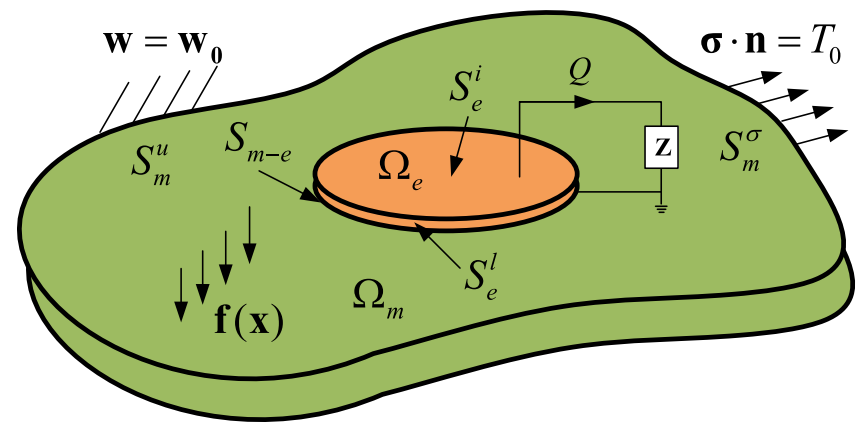

Figure 4. A generic piezo-mechanical system.

each row is fulfilled by choosing the negative capacitance value according to equation (6).

\section{Numerical model and energy analysis}

\subsection{Finite element model of the piezo-mechanical system}

The piezo-mechanical system studied in this paper can be generally illustrated by figure 4 . In this system, the mechanical structure occupies a domain $\Omega_{m}$ with particular Dirichlet boundary conditions applied on the surface $S_{m}^{u}$ and Neumann boundary conditions on the surface $S_{m}^{\sigma}$. A set of piezoelectric transducers are connected to the mechanical structure occupying a domian $\Omega_{e}$ (only one is depicted in the figure as an example). Zero charge conditions are applied to the lateral surfaces $S_{e}^{l}$ of the piezoelectric transducers. The connecting interfaces $S_{m-e}$ are grounded and the free electrode $S_{e}^{i}$ of each piezoelectric transducer is applied with a surface charger $\mathrm{Q}_{0}^{i}$ or a voltage $\mathrm{V}_{0}^{i}$, here $i \in[1,2, \ldots, P]$ and $P$ is the total number of the piezoelectric transducers.

The 3D dynamical equilibrium equations for the piezomechanical system described above can be written as:

$$
\begin{aligned}
\rho \ddot{\boldsymbol{w}}-\nabla \cdot \boldsymbol{\sigma} & =\boldsymbol{f}, \quad \forall \boldsymbol{x} \in \Omega_{m} \cup \Omega_{e} \\
\nabla \cdot \boldsymbol{D} & =0, \quad \forall \boldsymbol{x} \in \Omega_{e}
\end{aligned}
$$

with associated mechanical boundary conditions:

$$
\begin{array}{rlrl}
\boldsymbol{w} & =\boldsymbol{w}_{0}, & \forall \boldsymbol{x} \in S_{m}^{u} \\
\boldsymbol{\sigma} \cdot \boldsymbol{n} & =T_{0}, \quad \forall \boldsymbol{x} \in S_{m}^{\sigma}
\end{array}
$$

and electric boundary conditions:

$$
\begin{array}{rlrl}
\boldsymbol{D} \cdot \boldsymbol{n} & =0, & & \forall \boldsymbol{x} \in S_{e}^{l} \\
V & =0, & \forall \boldsymbol{x} \in S_{m-e} \\
\int \boldsymbol{D} \cdot \boldsymbol{n} \mathrm{d} s & =Q_{0}^{i} & & \text { or } \\
\varphi & =V_{0}^{i}, & & \forall \boldsymbol{x} \in S_{e}^{i} \text { and } i \in[1,2, \ldots, P] .
\end{array}
$$

In the equations above, $\boldsymbol{w}$ is the mechanical displacement tensor, $\boldsymbol{\sigma}$ is the stress tensor, $\boldsymbol{f}$ is the applied external force tensor, $\boldsymbol{D}$ is the electric displacement tensor and $\phi$ is the electric potential, $\boldsymbol{n}$ is the outward unit normal vector.

The stress tensor and the electric diplacement tensor in piezoelectric materials are related to the linear strain tensor $\varepsilon$ 
Table 1. Geometry parameters of one unit piezoelectric lattice.

\begin{tabular}{cccc}
\hline$l_{b}$ & $h_{b}$ & $l_{p}$ & $h_{p}$ \\
\hline $0.04 \mathrm{~m}$ & $0.005 \mathrm{~m}$ & $0.035 \mathrm{~m}$ & $0.001 \mathrm{~m}$ \\
\hline
\end{tabular}

and electric field tensor $\boldsymbol{E}$ through the constitutive relations below:

$$
\begin{aligned}
& \boldsymbol{\sigma}=\boldsymbol{C}_{E}: \varepsilon-\boldsymbol{e}^{T} \cdot \boldsymbol{E} \\
& \boldsymbol{D}=\boldsymbol{e} \cdot \varepsilon+\boldsymbol{\epsilon}_{S} \cdot \boldsymbol{E} .
\end{aligned}
$$

Here, $\boldsymbol{C}_{E}, \boldsymbol{e}$ and $\boldsymbol{\epsilon}_{S}$ denote the elasticity tensor at constant electric field, the piezoelectric coupling tensor and the dielectric permittivity tensor at constant strain, respectively. $(\cdot)^{T}$ indicates transposition. The strain tensor is calculated as $\varepsilon=\frac{1}{2}\left(\nabla \cdot \boldsymbol{w}+\nabla^{T} \cdot \boldsymbol{w}\right)$. The electric field tensor and the electric potential are related as $\boldsymbol{E}=-\nabla \varphi$.

Assume that the electric potentials on the free electrode of each piezoelectric transducer are identical. By using the finite element discretization technique, the discrete governing equations for the piezo-mechanical system are obtained:

$$
\begin{aligned}
\boldsymbol{M}_{d d} \ddot{\boldsymbol{d}}+\boldsymbol{K}_{d d} \boldsymbol{d}+\boldsymbol{K}_{d V} \boldsymbol{V} & =\boldsymbol{F} \\
\boldsymbol{K}_{d V}^{T} \boldsymbol{d}+\boldsymbol{K}_{V V} \boldsymbol{V} & =\boldsymbol{Q} .
\end{aligned}
$$

Here, $\boldsymbol{d}$ and $\boldsymbol{V}$ represent the structural and electric degree of freedom, respectively.

The negative capacitance circuit is introduced into each piezoelectric transducer by applying the following electric boundary condition:

$$
Q=C_{n e g} V .
$$

In the numerical simulations in this paper, the structures were discretized by 3D quadratic Lagrange elements. At least ten elements were guaranteed in one wavelength. The simulation domain was surrounded by perfectly matched layers to avoid wave reflections at the boundaries $[35,36]$. The piezolens in the simulations has dimensions of $0.24 \mathrm{~m} * 0.58 \mathrm{~m}$ in the $x-y$ plane as depicted in figure 1 . The geometry parameters for one unit of the piezoelectric lattice are illustrated in table 1; corresponding meanings of the symbols in the table can be found in figure 2. The Young's modulus, the Poisson's ratio and the density of the aluminum are $E_{b}=70 \mathrm{Gpa}$, $\mu_{b}=0.3$ and $\rho_{b}=2700 \mathrm{~kg} / \mathrm{m}^{3}$, respectively. The material parameters for the PZT26 are listed in table 2. Light damping amounting to a hysteretic coefficient of $0.1 \%$ was applied.

\subsection{Energy analysis for a harmonically excited thin plate}

To better understand the underlying physics and the focusing effects of the piezo-lens, energy analyses including power flow or kinetic energy have been conducted.

The very essential consequence of wave propagation is the power diffusion in the medium. The power diffusion pattern can be characterized by power flows. Accordingly, power flows are useful in depicting the wave paths inside the lens zone. In the frequency domain, the power flow in an elastic medium is defined as:

$$
\boldsymbol{I}=\frac{1}{2} \omega \cdot r e\left(-\boldsymbol{\sigma} \cdot \boldsymbol{w}^{*}\right) .
$$

Here, $(\cdot)^{*}$ represents the conjugate value and $r e(\cdot)$ means only the real part is retained. Therefore, the power flow in a thin plate excited by harmonic forces can be obtained as:

$$
\boldsymbol{I}_{\text {tot }}=\int_{-\frac{h}{2}}^{\frac{h}{2}} \boldsymbol{I} \mathrm{d} z
$$

For harmonically excited structures, the time averaged kinetic energy is:

$$
W_{k}=\frac{1}{4} \omega^{2} \rho \boldsymbol{w}^{* T} \cdot \boldsymbol{w} .
$$

It will be used in estimating the focusing effects.

\section{Numerical results}

\subsection{Focusing effect}

In the simulations, to verify the focusing effect of the piezolens, flexural waves were excited by a surface line harmonic transverse force located $0.1 \mathrm{~m}$ away from the left boundary of the lens. The parameters of the piezo-lens were set as $\alpha=\pi / 0.6$ and $\beta=0$. With these settings, theoretically flexural waves will be focused at a distance $f=0.3 \mathrm{~m}$ on the $y=0$ line.

Figure 5 shows the normalized power flows in the host plate without and with a piezo-lens at $2000 \mathrm{~Hz}$. In the figure, the arrows indicate the directions and magnitude of the power

Table 2. Material parameters of PZT26.

\begin{tabular}{lcc}
\hline Symbol & Value & Property \\
\hline$S_{11}^{E}=S_{22}^{E}, S_{33}^{E}$ & $1.30 \mathrm{E}-11,1.96 \mathrm{E}-11\left(\mathrm{~Pa}^{-1}\right)$ & Compliance matrix under constant electric field \\
$S_{12}^{E}, S_{13}^{E}=S_{23}^{E}$ & $-4.35 \mathrm{E}-12,-7.05 \mathrm{E}-12\left(\mathrm{~Pa}^{-1}\right)$ & \\
$S_{44}^{E}=S_{55}^{E}, S_{66}^{E}$ & $3.32 \mathrm{E}-11,3.47 \mathrm{E}-11\left(\mathrm{~Pa}^{-1}\right)$ & \\
$d_{31}=d_{32}$ & $-1.28 \mathrm{E}-10(C / N)$ & Piezoelectric matrix \\
$d_{33}$ & $3.28 \mathrm{E}-10(C / N)$ & \\
$d_{24}=d_{15}$ & $3.27 \mathrm{E}-10(C / N)$ & Density \\
$\rho$ & $7700\left(\mathrm{~kg} / \mathrm{m}^{3}\right)$ & Dielectric permittivity under constant stress \\
$\varepsilon_{11}^{\sigma}=\varepsilon_{22}^{\sigma}, \varepsilon_{33}^{\sigma}$ & $1.19 E+03 \varepsilon_{0}, 1.33 E+03 \varepsilon_{0}$ & \\
\hline
\end{tabular}




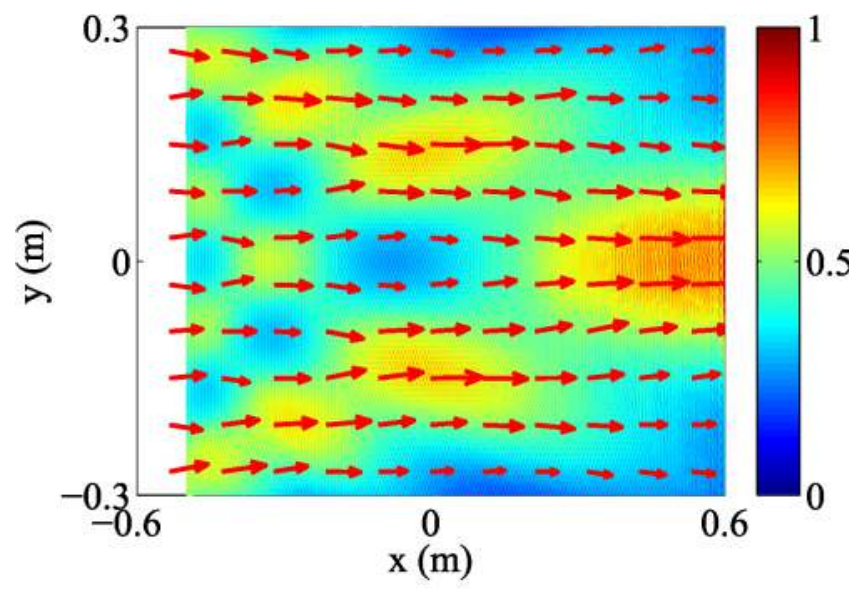

(a) without piezo-lens

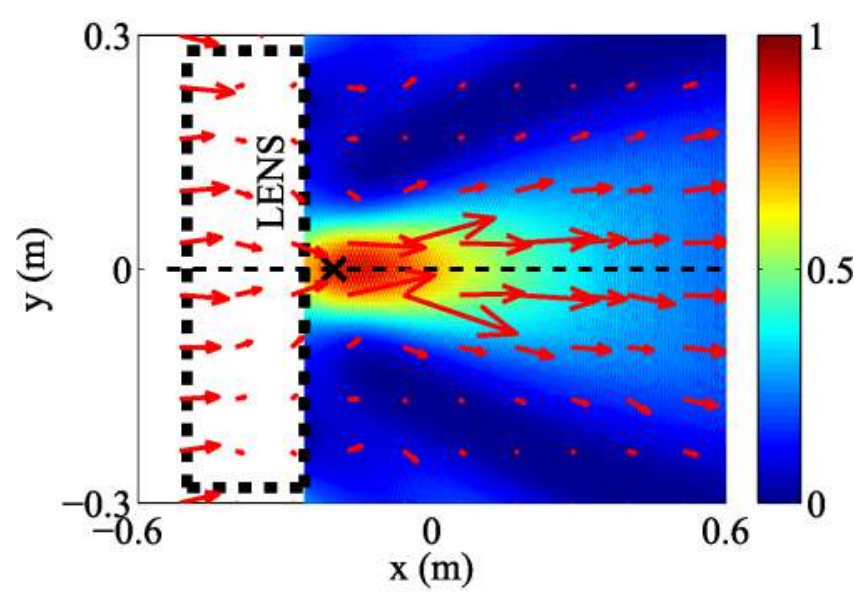

(b) with piezo-lens

Figure 5. Normalized power flows in the host plate at $2000 \mathrm{~Hz}$ (a) without and (b) with the piezo-lens. The black cross indicates the designed focal point.

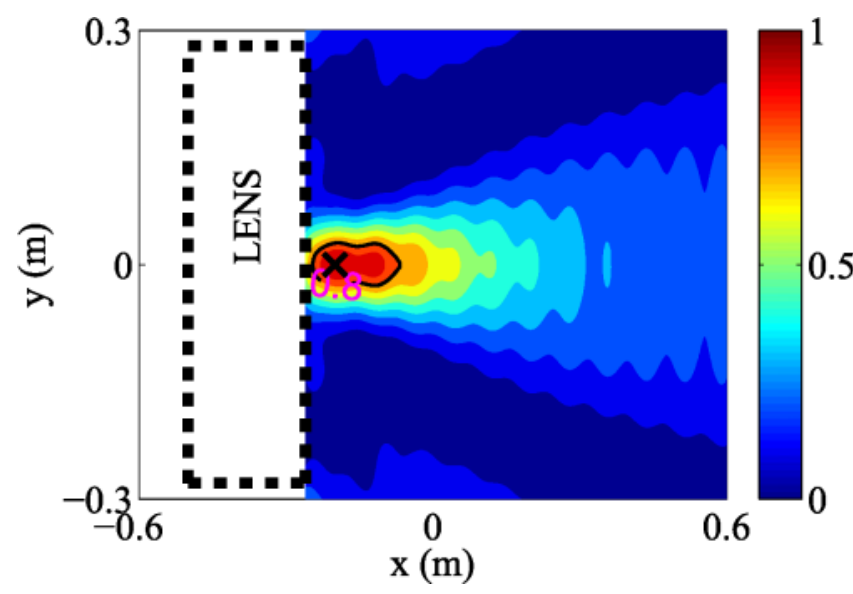

Figure 6. Normalized kinetic energy distribution after the piezo-lens at $2000 \mathrm{~Hz}$. The black cross indicates the designed focal point, the solid line indicates the energy concentration zone.

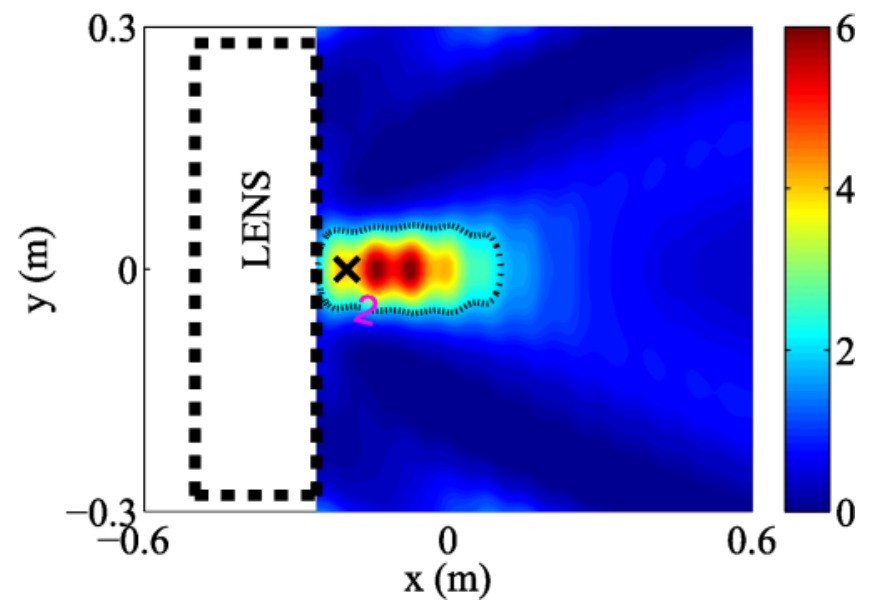

Figure 7. Energy enhancement ratio distribution after the piezo-lens at $2000 \mathrm{~Hz}$. The black cross indicates the designed focal point, the dashed line indicates the energy enhancement zone.

flows. It can be observed that the magnitude of the power flows in the plate reduces when the waves are incident into the piezo-lens zone, and the reduction becomes more obvious at locations far from the symmetry axis $y=0$. There are two reasons for this phenomenon. On one hand, the refractive indexes inside the piezo-lens are designed to fit equation (1). Thus, at the left and right interfaces between the piezo-lens and the background plate, the impedances only match on the symmetry axis, and the impedance discontinuities increase with the distance away from the symmetry axis. As a result, part of the incident waves will be reflected at the interfaces and the reflections gain with the distance away from the symmetry axis. On the other hand, in the piezo-lens zone, the piezoelectric patches are bonded on the surfaces of the host plate, and part of the incident power in the host plate will flow into these patches. It can also be observed that, except near the upper and lower boundaries, power inside the piezo-lens zone flows toward the designed focal point. This verifies that the piezo-lens bends the flexural waves as designed. It should be noted that the outgoing power near the upper and lower boundaries is not unique to the piezo-lens-it is a characteristic of the flat GRIN lens; more details can be found in the supplementary document stacks.iop.org/sms/25/075007/ mmedia.

Figure 6 is the normalized kinetic energy distribution pattern after the piezo-lens at $2000 \mathrm{~Hz}$. It can be observed that due to the wave bending effect, most of the incident energy is concentrated inside a limited zone around the designed focal point. To characterize this energy concentration effect, an energy concentration zone is defined. The kinetic energy inside this zone satisfies the following condition:

$$
\frac{W_{k}}{\max \left(W_{k}\right)} \geqslant 0.8
$$



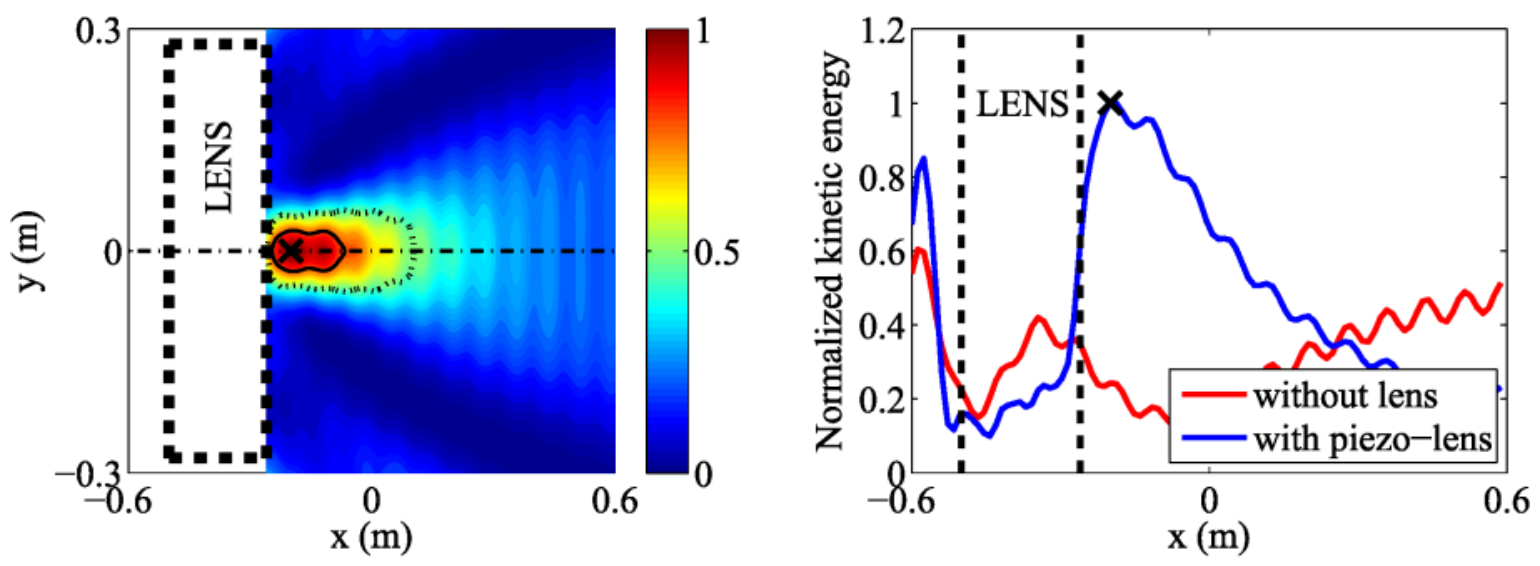

(a) $\alpha=\pi / 0.6, \beta=0$
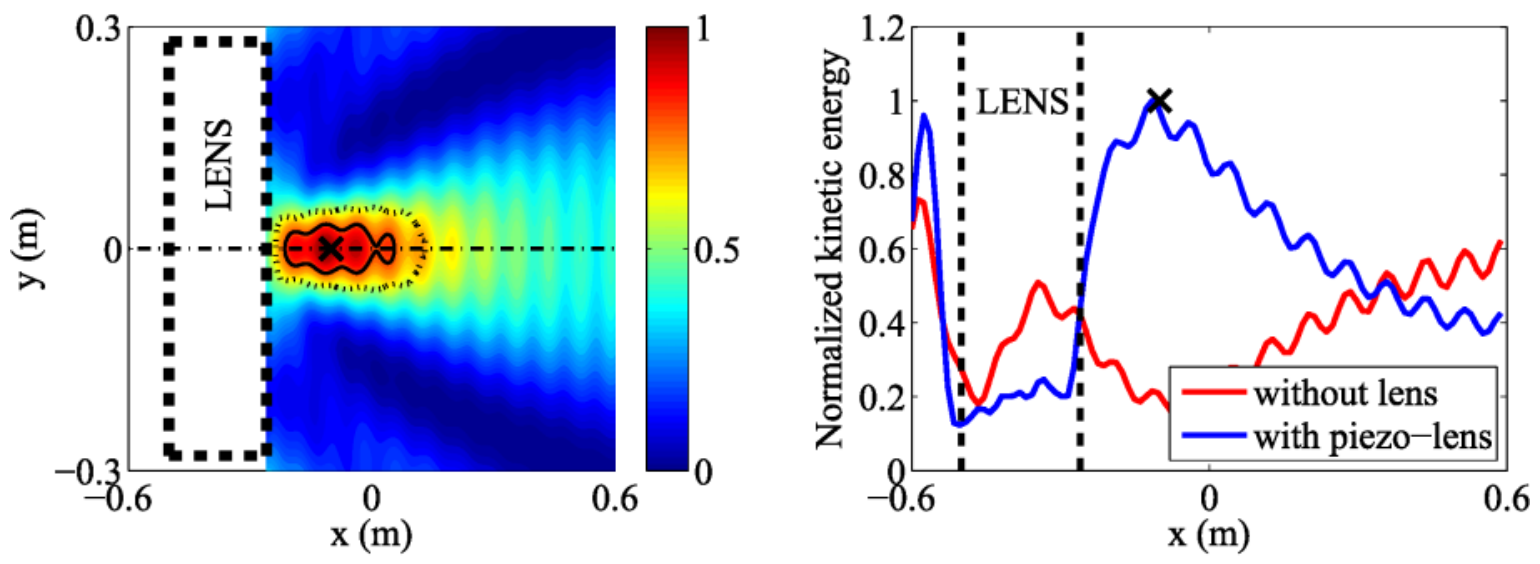

(b) $\alpha=\pi / 0.8, \beta=0$
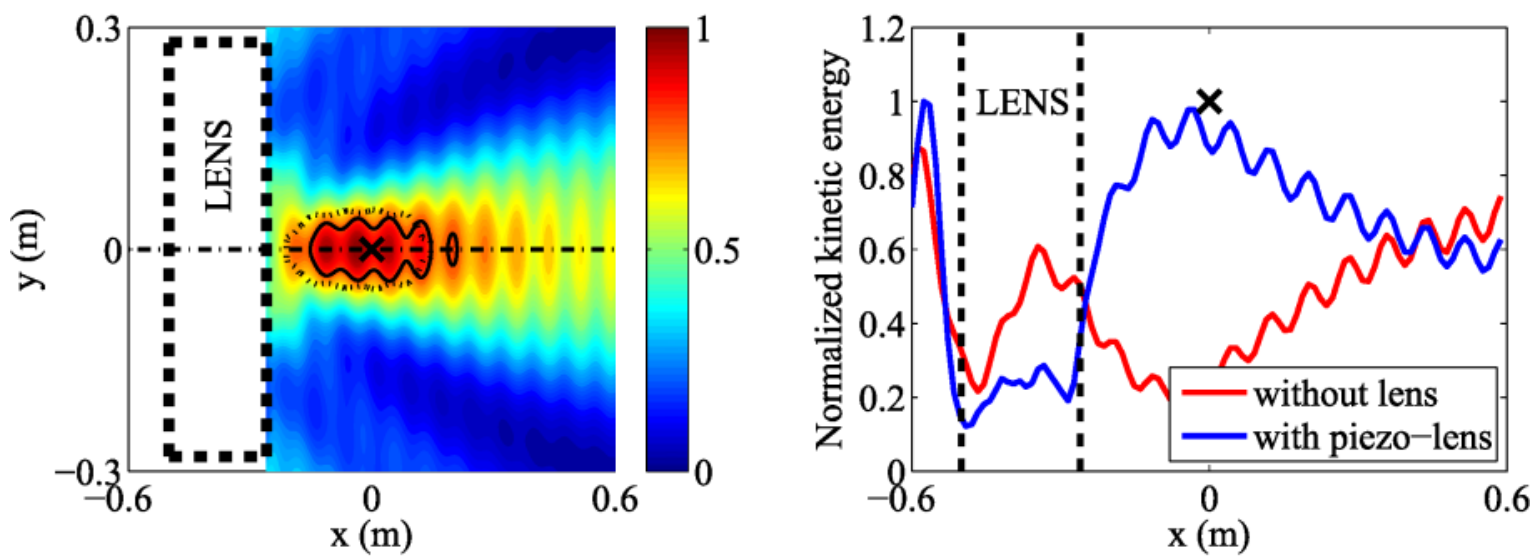

(c) $\alpha=\pi, \beta=0$

Figure 8. Left panel: normalized kinetic energy distributions after the piezo-lens for different focal locations at $2000 \mathrm{~Hz}$. The black crosses indicate the designed focal points, the solid and dashed lines indicate the energy concentration zones and the energy enhancement zones, respectively. Right panel: normalized kinetic energy along $y=0$ lines for different focal locations at $2000 \mathrm{~Hz}$. The black crosses indicate the designed focal points.

The condition in equation (16) indicates that inside the energy concentration zone, the kinetic energy is larger than or equal to 0.8 times the maximum kinetic energy after the lens. This zone is highlighted by a solid line in this paper. For example, the energy concentration zone at $2000 \mathrm{~Hz}$ is illustrated in figure 6.

Compared to the case without the lens, the energy concentration effect after the piezo-lens could enhance the energy 

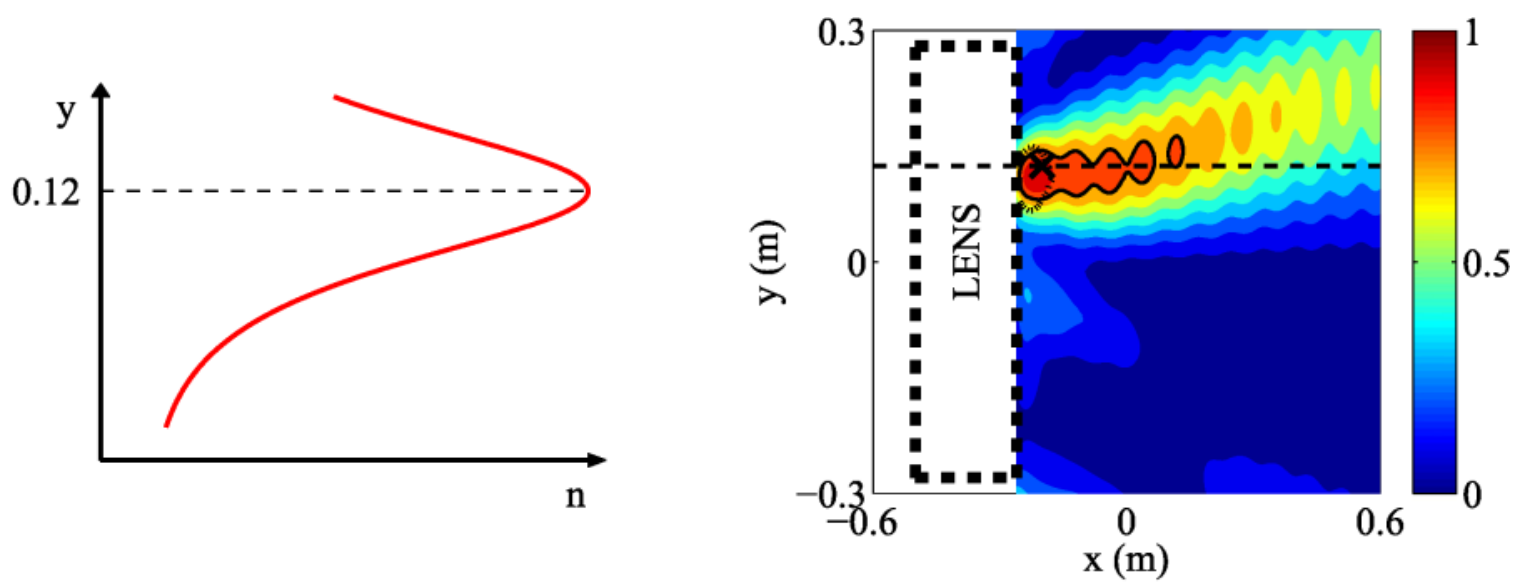

(a) $\alpha=\pi / 0.6, \beta=0.12$
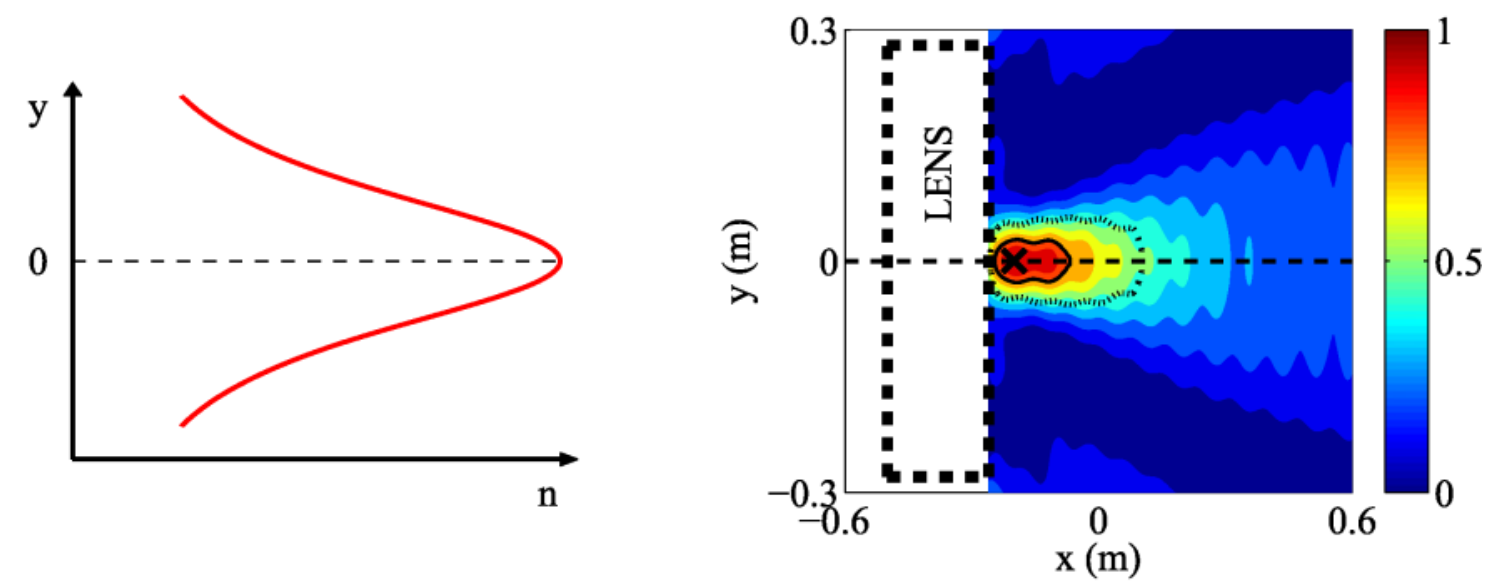

(b) $\alpha=\pi / 0.6, \beta=0$
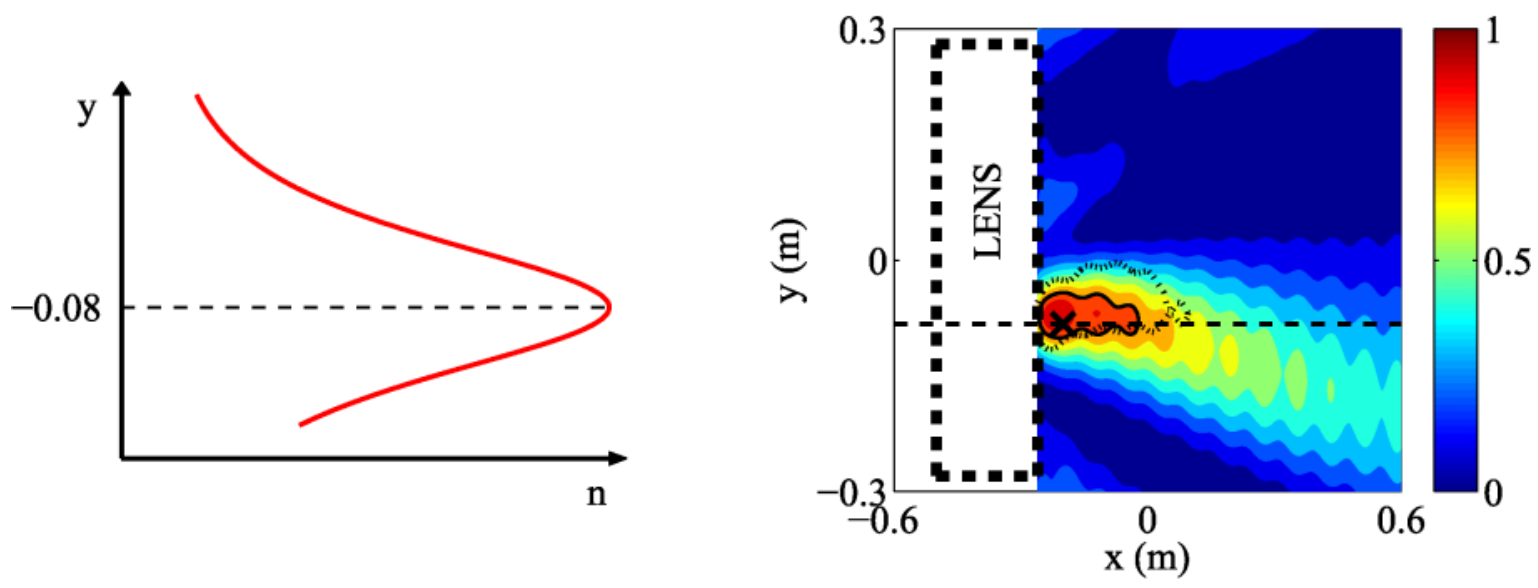

(c) $\alpha=\pi / 0.6, \beta=-0.08$

Figure 9. Left panel: designed refractive index profiles. Right panel: normalized kinetic energy distributions after the piezo-lens for different refractive index profiles at $2000 \mathrm{~Hz}$. The black crosses indicate the designed focal points, the solid and dashed lines indicate the energy concentration zones and the energy enhancement zones, respectively. 

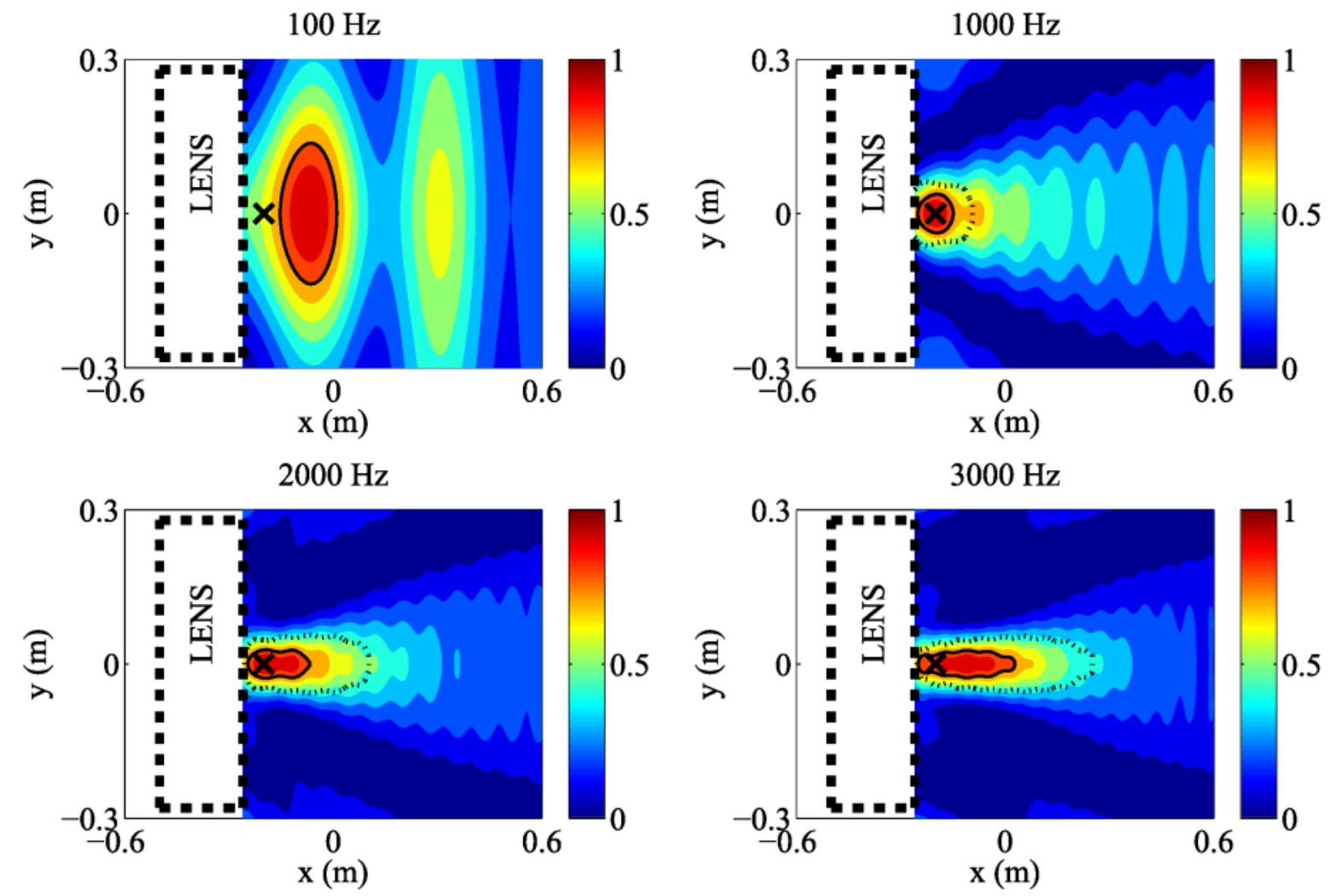

$4000 \mathrm{~Hz}$
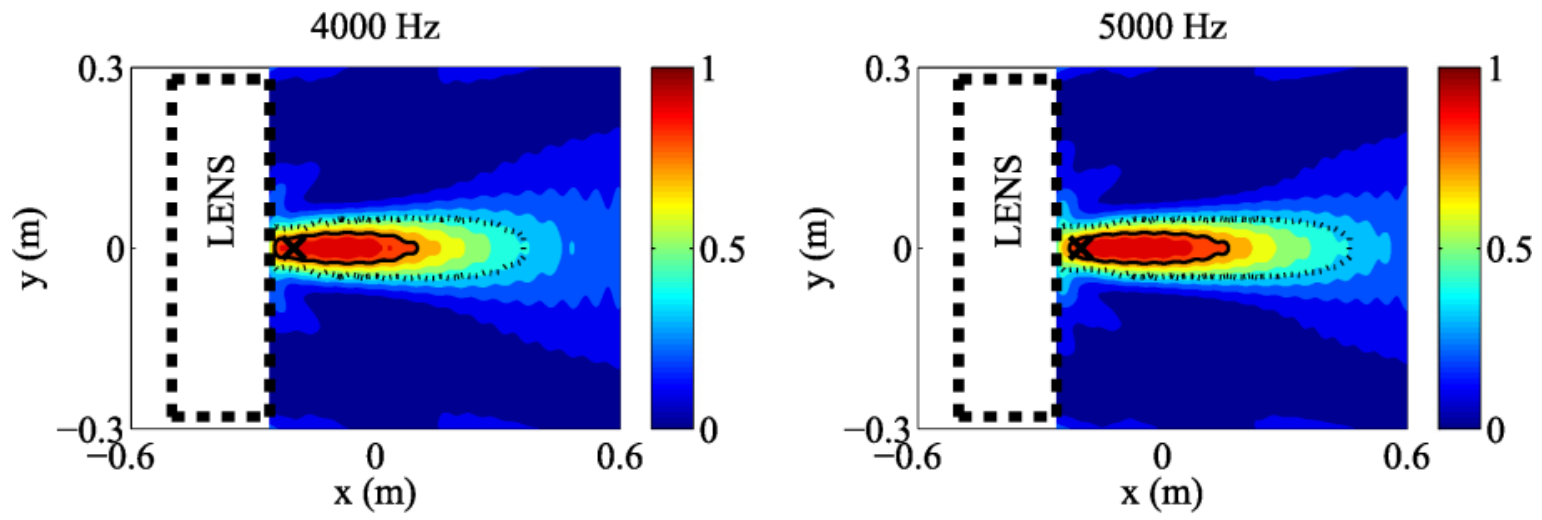

$6000 \mathrm{~Hz}$
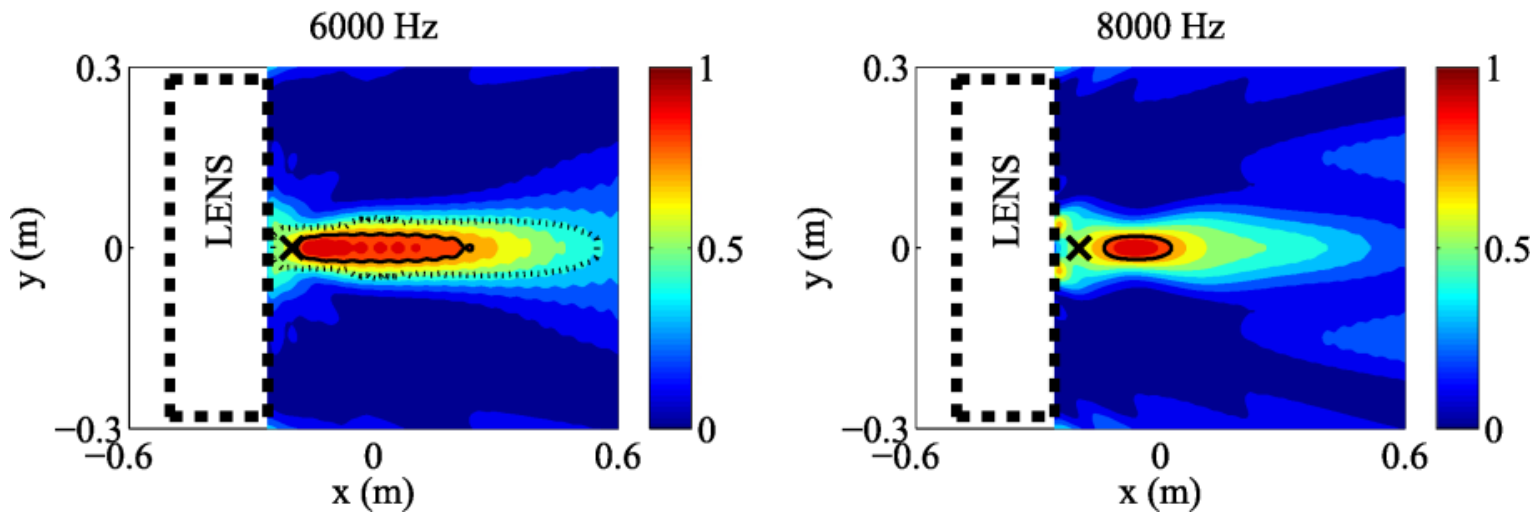

Figure 10. Normalized kinetic energy distributions after the piezo-lens at different frequencies. Black crosses indicate the designed focal points, solid and dashed lines indicate the energy concentration zones and the energy enhancement zones, respectively. 


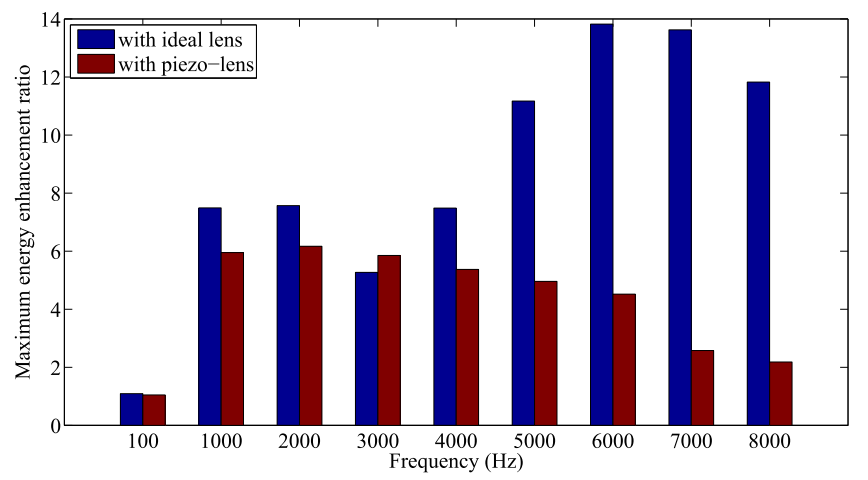

Figure 11. The maximum energy enhancement ratios at different frequencies.

Table 3. Geometry parameters of one unit piezoelectric lattice in the new piezo-lens.

\begin{tabular}{cccc}
\hline$l_{b}$ & $h_{b}$ & $l_{p}$ & $h_{p}$ \\
\hline $0.03 \mathrm{~m}$ & $0.005 \mathrm{~m}$ & $0.025 \mathrm{~m}$ & $0.001 \mathrm{~m}$ \\
\hline
\end{tabular}

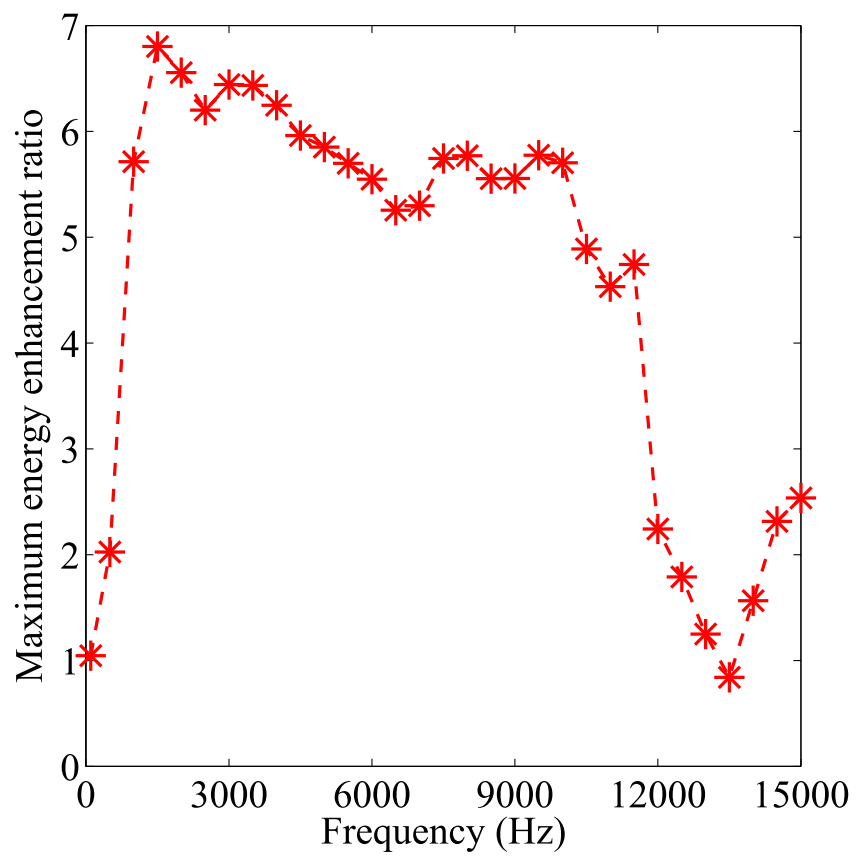

Figure 12. The maximum energy enhancement ratios at different frequencies for the new piezo-lens with smaller lattices.

in the particular zone. To illustrate this effect, an energy enhancement ratio (Eer) is defined as the ratio of the kinetic energy of the plate with the lens to the kinetic energy of the plate without the lens:

$$
\operatorname{Eer}(x, y)=\frac{W_{k}^{l}(x, y)}{W_{k}(x, y)} .
$$

In figure 7, the Eer after the piezo-lens at $2000 \mathrm{~Hz}$ is illustrated. According to figures 6 and 7, it can be observed that the energy is significantly enhanced inside the energy concentration zone. Similarly, to characterize this energy enhancement effect, an energy enhancement zone is defined. In this zone, the Eer satisfies the following condition:

$$
\operatorname{Eer}(x, y) \geqslant 2 \text {. }
$$

The energy enhancement zone is depicted by a dashed line in this paper, as can be observed in figure 7 .

\subsection{Adaptive ability}

In the piezo-lens, different refractive index profiles can be fulfilled by just tuning the shunting negative capacitance values, i.e. with the same geometry configuration, the piezolens can focus waves at different locations. This adaptive ability is demonstrated here, and in these simulations the flexural waves are excited by a surface line harmonic transverse force located $0.1 \mathrm{~m}$ away from the left boundary of the lens.

The piezo-lens can focus waves at different locations in the $x$ direction. To verify this, the parameter $\beta$ is fixed as $\beta=0$, but the parameter $\alpha$ is chosen as $\alpha=\pi / 0.6$, $\alpha=\pi / 0.8$ and $\alpha=\pi$, to focus waves at distances $0.3 \mathrm{~m}$, $0.4 \mathrm{~m}$ and $0.5 \mathrm{~m}$ on the $y=0$ line, respectively. The focusing effect of these three piezo-lenses at $2000 \mathrm{~Hz}$ is illustrated in figure 8. In the left panel, the normalized kinetic energy distribution after the piezo-lenses are shown. Depicted in the right panel are the normalized kinetic energy of the plates with the piezo-lenses along the $y=0$ line; the results of the plates without a lens are also illustrated as references. The adaptive ability of the piezo-lens in the $x$ direction can be observed from the results in figure 8 . It can also be observed that the energy concentration zone enlarges as the focal length increases, indicating that the energy will be less concentrated at a larger distance.

The piezo-lens can also focus waves at different locations in the $y$ direction. For example, fix the parameter $\alpha$ as $\alpha=\pi / 0.6$ and chose the parameter $\beta$ as $\beta=0.12, \beta=0$ and $\beta=-0.08$ to focus waves at a distance $0.3 \mathrm{~m}$ on the $y=0.12$ line, $y=0$ line and $y=-0.08$ line, respectively. Figure 9 shows the designed refractive index profiles and the focusing effects of the corresponding piezo-lenses at $2000 \mathrm{~Hz}$. It can be observed that the piezo-lens is adaptive in the $y$ direction. It should be noted that a larger distance between the symmetry axis of the refractive index profile $(y=\beta$ line $)$ and the central axis of the lens $(y=0$ line) will result in less energy concentration intensity and a smaller energy enhancement zone, as illustrated in the figure.

\subsection{Performances of the piezo-lens at different frequencies}

The focusing effects of the piezo-lens at different frequencies are studied in this sub-section. In the numerical simulations, the same excitation source and parameters of the piezo-lens used in the simulations in sub-section 4.1 were adopted.

Figure 10 shows the focusing effects of the piezo-lens at different frequencies. As expected, the effectiveness of the piezo-lens is limited within a certain frequency band. The 
$1000 \mathrm{~Hz}$

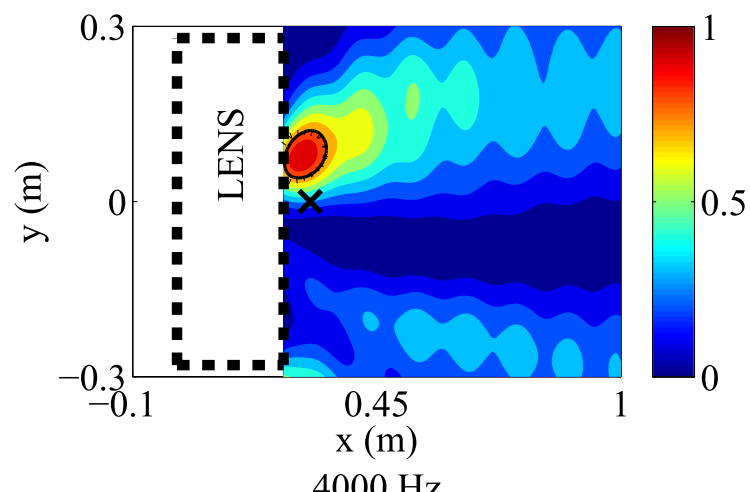

$4000 \mathrm{~Hz}$

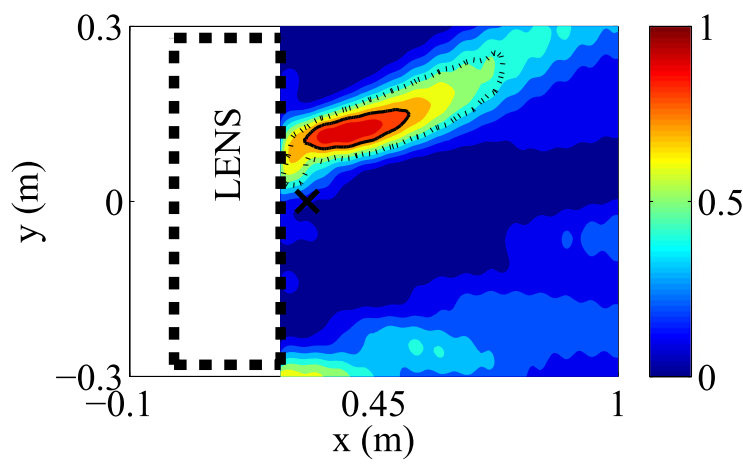

$2000 \mathrm{~Hz}$
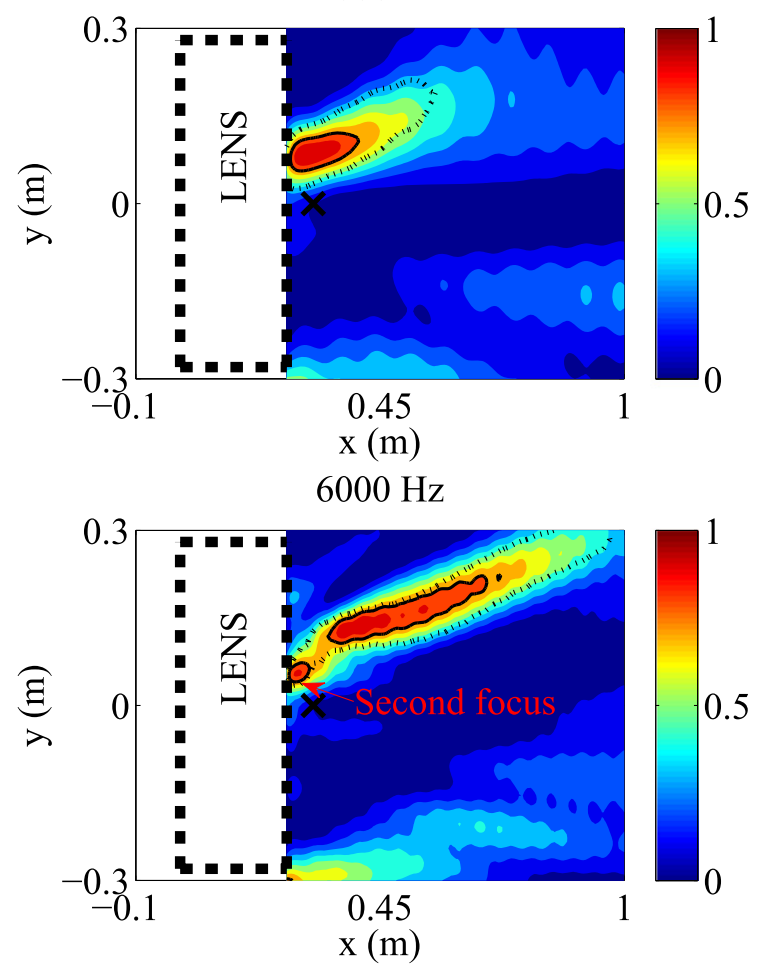

Figure 13. Normalized kinetic energy distributions after the piezo-lens for waves incident with $\theta=20^{\circ}$. The black cross represents the designed focal point, the solid and dashed lines indicate the energy concentration zones and the energy enhancement zones, respectively.

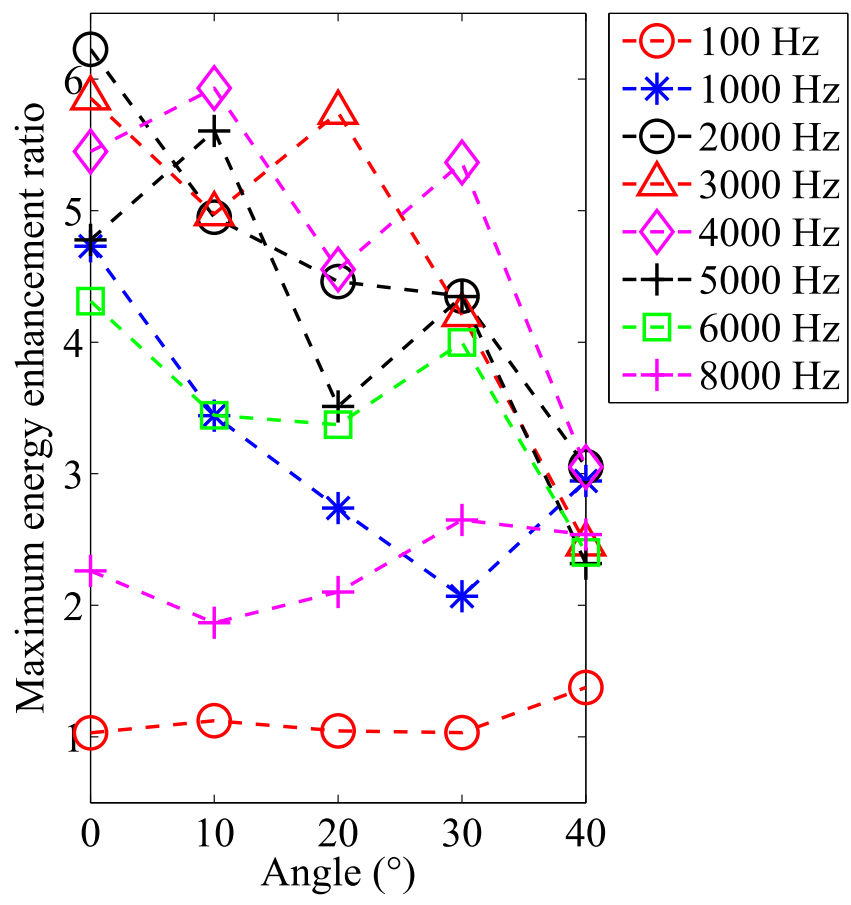

Figure 14. Variation of the maximum energy enhancement ratio with the incident angle at different frequencies.

lower limit frequency is dominated by the characteristic length of the piezo-lens. At frequencies smaller than the lower limit, the corresponding wavelengths will be larger than the characteristic length of the piezo-lens. Under these circumstances, waves will bypass the piezo-lens by a diffraction effect [2] and the piezo-lens will have a poor focusing performance. The lower limit for the piezo-lens in this paper is around $100 \mathrm{~Hz}$. At this frequency, the flexural wavelength is just a little longer than the piezo-lens' length in the $y$ direction. As a result of the diffractive effect, most of the waves will bypass the piezo-lens without being focused, leading to quite a large energy concentration zone and no energy enhancement zone. On the other hand, the upper limit frequency is dominated by the length of the lattice. At frequencies near the upper limit, the flexural wavelengths will be approximately equal to twice the lattice's length. Most of the incident energy will be reflected by the piezo-lens [29], which results in poor performance. The upper limit in this paper is about $8000 \mathrm{~Hz}$. At $8000 \mathrm{~Hz}$, the wavelength is almost equal to twice the lattice's length. At this frequency, even though an energy concentration zone can still be observed, there is no energy enhancement zone due to the large reflection.

Inside the effective frequency band, the performances of the piezo-lens are dependent on the frequencies. At frequencies from $1000 \mathrm{~Hz}-6000 \mathrm{~Hz}$, as the frequency increases, energy will be less concentrated and the energy concentration zone will shift to the right-hand side of the designed focal point. The consequent energy enhancement zone will become longer in the $x$ direction. However, below $2000 \mathrm{~Hz}$, energy is concentrated almost around the designed focal point. The wavelength at $2000 \mathrm{~Hz}$ is nearly four times the lattice's length. Hence, the piezo-lens could focus energy near the designed point if the wavelength is larger than four times the lattice's length. 


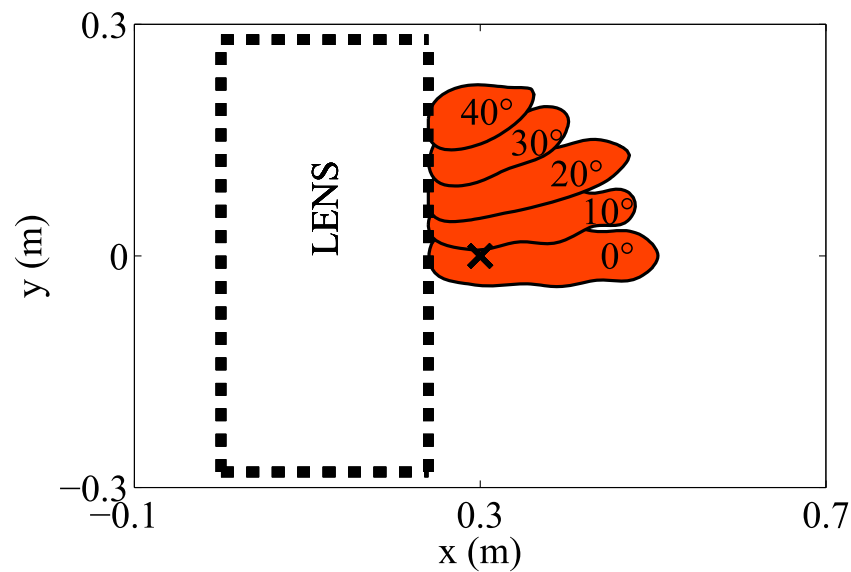

(a) energy concentration zone

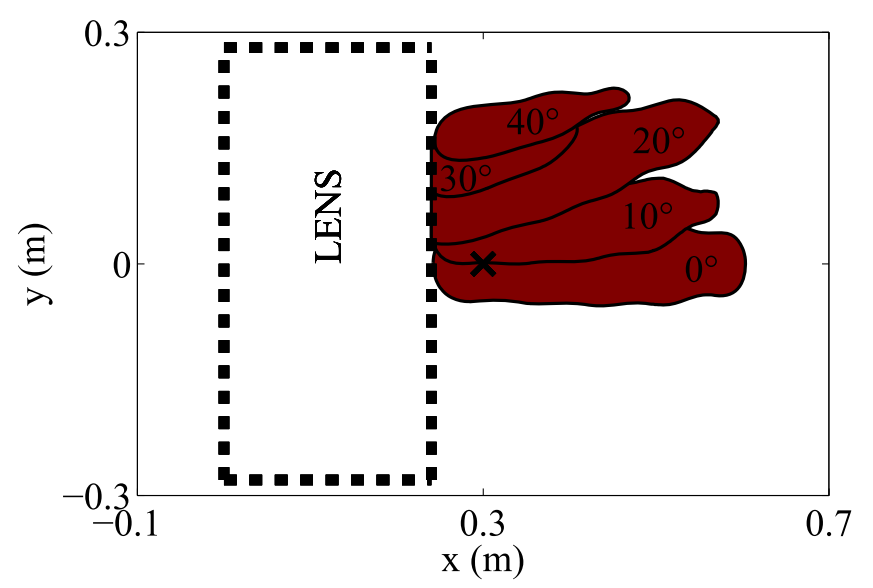

(b) energy enhancement zone

Figure 15. Influence of the incident angle on (a) the energy concentration zone and (b) the energy enhancement zone at $2000 \mathrm{~Hz}$. The black cross represents the designed focal point.

There are several reasons that probably contribute to the shifting of the focal location and less concentration of energy at higher frequencies inside the effective frequency band. Firstly, the wavelength is more comparable to the lattice's length at higher frequencies. In the piezo-lens, the variation of the refractive index is realized in a piecewise form. At smaller wavelengths, this variation will be less smooth. Secondly, the homogenized model of the piezoelectric lattice used in the designing process becomes less accurate at higher frequencies [33]. Accordingly, the actual refractive index variation profile in the piezo-lens will deviate from the designed one at these frequencies. Since the focal location of the waves is dependent on the refractive index profile inside the piezo-lens, this deviation will make the flexural waves focus away from the designed point. Thirdly, the anisotropy of the piezoelectric lattice could be more obvious at higher frequencies. The anisotropy of the lattice will cause the aberration of focus [37].

The performances of the piezo-lens at different frequencies can also be predicted by the maximum energy enhancement ratio $(\max (E e r))$ after the lens. The $\max ($ Eer $)$ after an ideal lens and a piezo-lens at different frequencies are illustrated in figure 11. The ideal lens is used as a reference. It has the same dimensions as the piezo-lens and is designed by consecutively varying the Young's modulus in the lens zone. In the figure, a larger $\max (E e r)$ generally indicates a better focusing effect. This can be seen from the fact that at the lower limit frequency $(100 \mathrm{~Hz})$ and at the upper limit frequency $(8000 \mathrm{~Hz})$, the $\max (E e r)$ after the piezo-lens are much smaller than most of those at frequencies inside the effective frequency band. As the frequency approaches the upper limit, the performance of the piezo-lens declines. This is coincident with the results revealed in figure 10. In particular, near the upper limit at $7000 \mathrm{~Hz}$, the performance of the piezo-lens is already poor. Therefore utilization near the upper limit should be avoided for better applications. The piezo-lens is commonly less effect than the ideal lens inside the effective frequency band. This is reasonable since the piezo-lens has a discrete configuration, and it will reflect more energy than the ideal one.

According to the results above, the effective frequency band of the piezo-lens can be extended by increasing the length of the piezo-lens and/or decreasing the length of the lattice. To demonstrate this, a new piezo-lens model with smaller lattices was designed. The new piezo-lens is composed of 19-by-8 array of piezoelectric lattices, each unit piezoelectric lattice has the dimensions illustrated in table 3 . The new piezo-lens has smaller lattices but has more, therefore there are little differences between the global dimensions of the new piezo-lens $(0.24 \mathrm{~m} * 0.57 \mathrm{~m})$ and the existing one $(0.24 \mathrm{~m} * 0.56 \mathrm{~m})$. The parameters of the new piezo-lens are set as $\alpha=\pi / 0.6$ and $\beta=0$, which are coincident with the settings in other simulations in this sub-section. Figure 12 shows the maximum energy enhancement ratio after the new piezo-lens at different frequencies. Compared with the results in figure 11 , it is clear that the effective frequency band has been significantly broadened, from $[1000,6000] \mathrm{Hz}$ to $[1000,11500] \mathrm{Hz}$.

\subsection{Performances of the piezo-lens for flexural waves exicited by different types of sources}

Flat GRIN lenses are originally designed to focus waves incident from the normal direction. Actually, any wave can be decomposed to have a component in the normal direction. Thus, a flat GRIN lens should have focusing effects for other kinds of incident waves to some extent. In this sub-section, the performances of the piezo-lens for obliquely incident plane waves and waves excited by a point source are studied. The parameters of the piezo-lens are set as $\alpha=\pi / 0.6$ and $\beta=0$ in this sub-section.

4.4.1. Oblique plane waves. In these cases, oblique plane waves are generated by a surface line harmonic transverse force with an angle $\theta$ against the left boundary of the piezolens. A positive $\theta$ represents waves that are incident from the bottom left direction and a negative one represents waves that 
$1000 \mathrm{~Hz}$

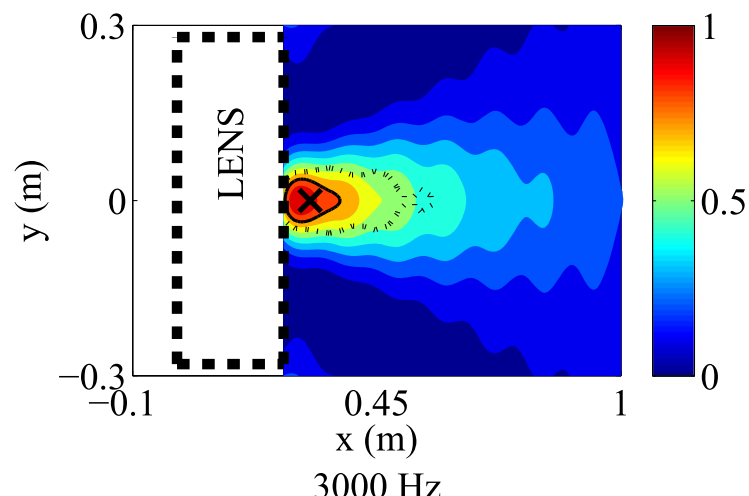

$3000 \mathrm{~Hz}$

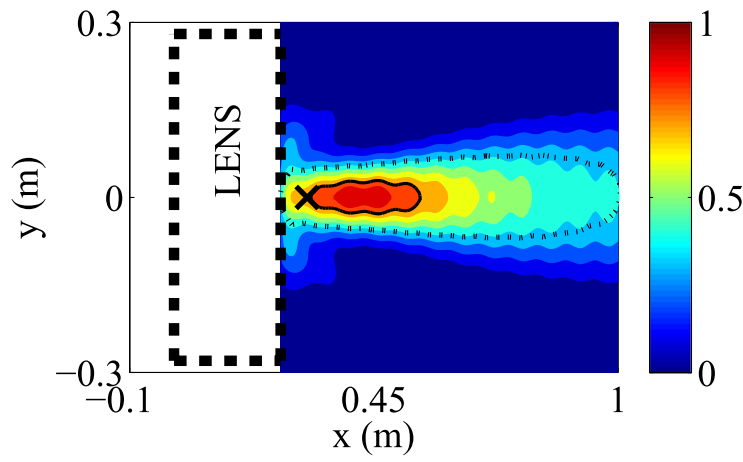

$2000 \mathrm{~Hz}$
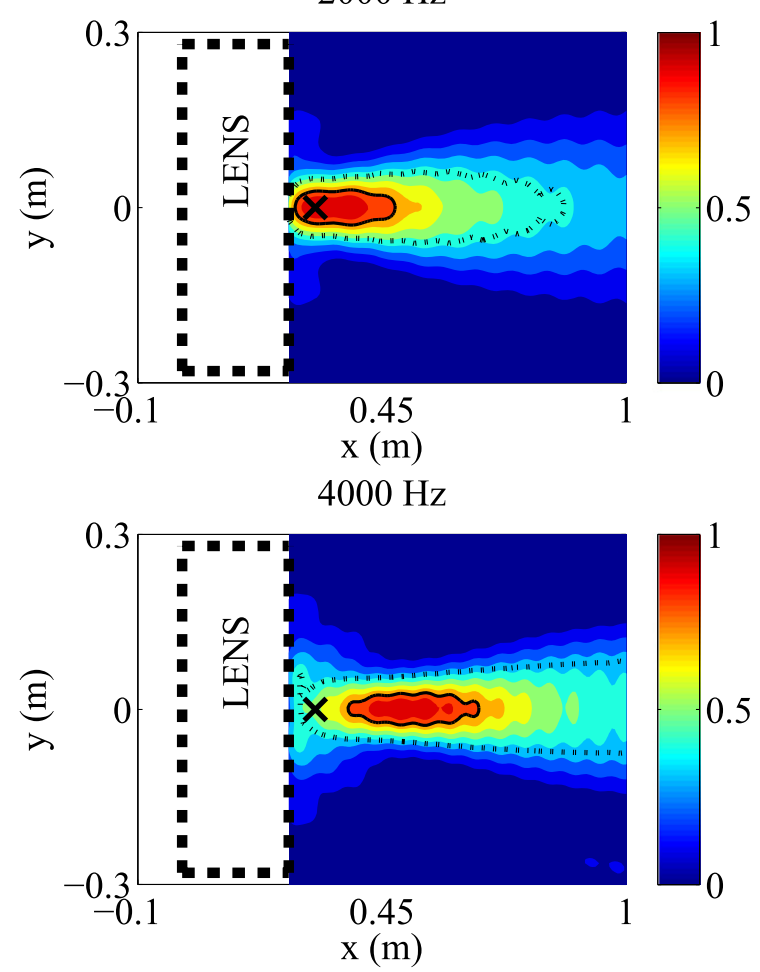

Figure 16. Normalized kinetic energy distributions after the piezo-lens for waves excited by a point force located $1.2 \mathrm{~m}$ away from the left lens boundary. The black crosses indicate the designed focal points, the solid and dashed lines indicate the energy concentration zones and the energy enhancement zones, respectively.

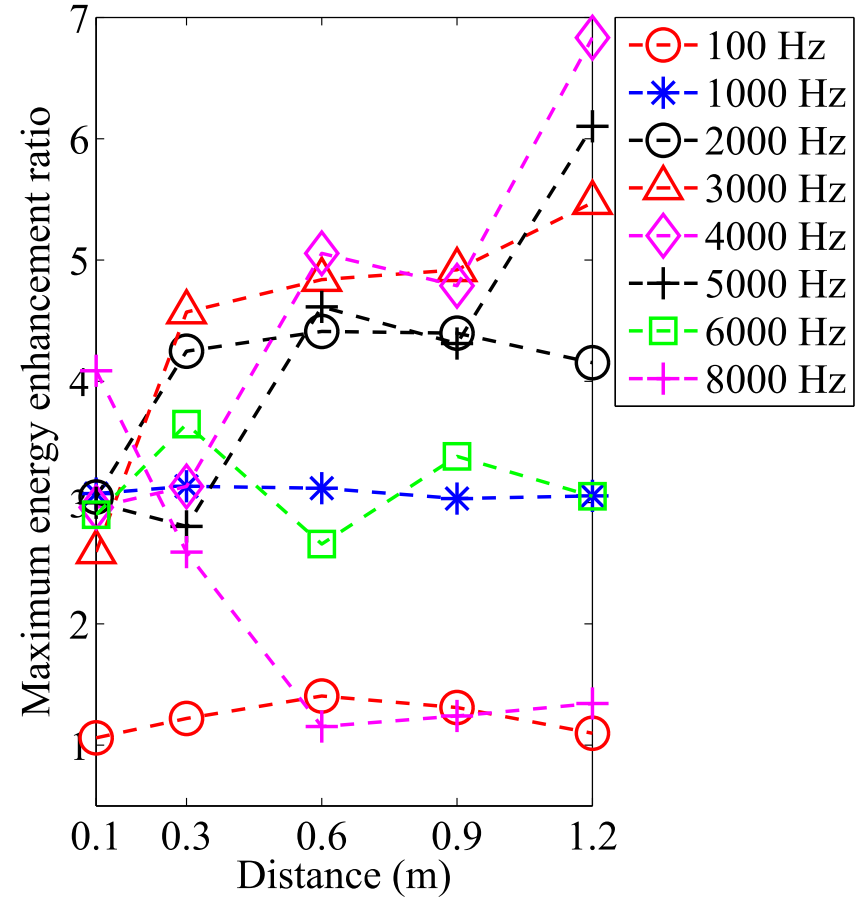

Figure 17. Influence of the point force distance on the maximum energy enhancement ratio at different frequencies.

are incident from the upper left direction; due to the symmetry, only the positive $\theta$ will be considered.

The focusing effects of these cases are demonstrated by an example in figure 13. For the oblique plane wave, energy is focused above the designed focal point. The deviation of the energy concentration zone from the designed location is expected. The wave incident with an angle can be decomposed into an $x$ component and a $y$ component. The $x$ component will be focused to a point and the $y$ component will deviate this real focal point away from the designed one in the $y$ direction. Similar to the normal incident wave case, as the frequency increases, the energy concentration zone will shift to the right and enlarge. Note that at a higher frequency $(6000 \mathrm{~Hz})$, an extra focalization can be observed near the right piezo-lens boundary. The reason for this phenomenon is interpreted in the supplementary document.

The influence of the incident angle on the focusing effects at different frequencies are predicted in figure 14. Inside the effective frequency band (from $1000 \mathrm{~Hz}-6000 \mathrm{~Hz}$ ), the overall trend is that the focusing effects decline with the increase of the incident angle. Particularly at $\theta=40^{\circ}$ case, the $\max (E e r)$ at frequencies inside the effective frequency band are comparable with that at the upper limit frequency (8000 $\mathrm{Hz})$. The $\max (\operatorname{Eer})$ at frequencies inside the effective frequency band should be much larger than that at limit frequencies if the piezo-lens has a good performance. From this point of view, the piezo-lens already has a poor effect at $\theta=40^{\circ}$ case. Therefore, the incident angle of the plane waves should be limited in a range-in our case here the range is about $\left[-40^{\circ}, 40^{\circ}\right]$.

The more specific influences of the incident angle on the focusing effects at $2000 \mathrm{~Hz}$ are illustrated in figure 15 . As the incident angle increases, the energy concentration zone will 


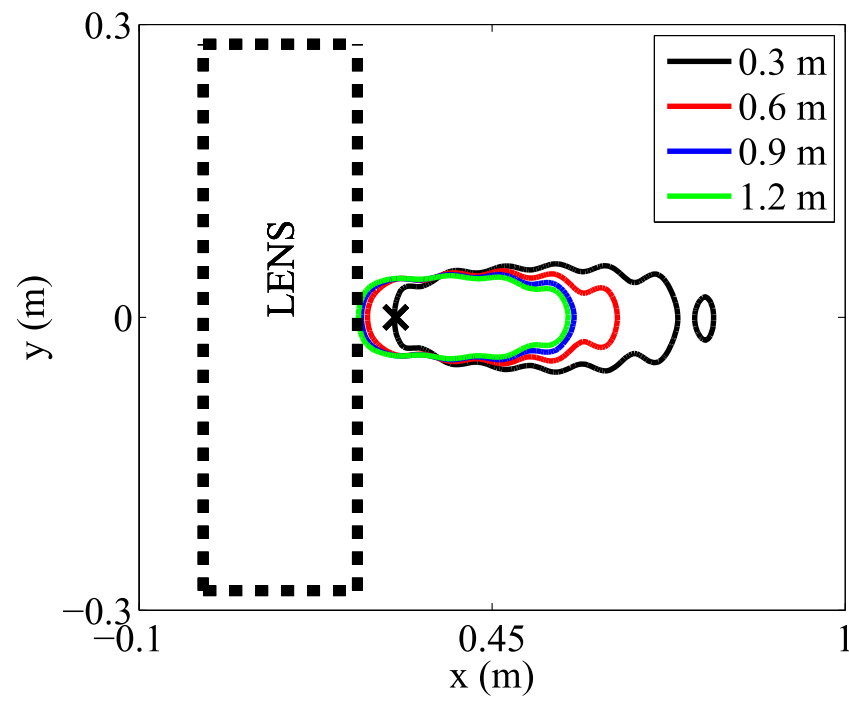

(a) energy concentration zone

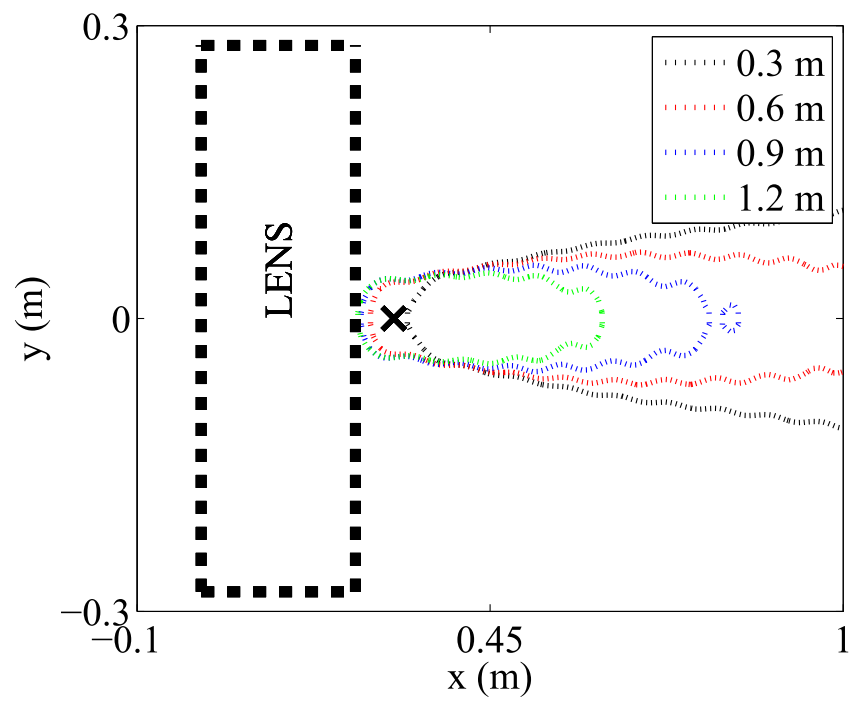

(b) energy enhancement zone

Figure 18. Influence of the point force distance on (a) the energy concentration zone and (b) the energy enhancement zone at $2000 \mathrm{~Hz}$. The black cross represents the designed focal point.

shift upward and become smaller. Consequently, the energy enhancement zone also shifts upward and shrinks. According to figures 14 and 15, at the case with larger incident angle, even though the energy is more concentrated, the $\max (E e r)$ at that case is smaller. This is mainly caused by the fact that more energy will be reflected when the waves are incident with a larger angle.

4.4.2. Waves excited by point forces. In these simulations, flexural waves are generated by a point force located on the central axis of the piezo-lens $(y=0$ line) with a distance $d$ from the left boundary of the piezo-lens. It is easy to understand that if the point force is far enough away from the piezo-lens, the waves incident upon the piezo-lens can be treated as plane waves; the piezo-lens should have focusing effects at these cases. For the $d=1.2 \mathrm{~m}$ case, the distance is more than five times the longest wave length of the considered frequency; it is assumed that this distance is far enough. The focusing effects at this case are illustrated in figure 16. It can be seen that energy is effectively focused. Comparing the corresponding results in figures 10 and 16, the right shifting of the energy concentration zone is more obvious in figure 16, indicating that the piezo-lens is more sensitive to frequency in the point force case.

The influence of point force distance on the focusing effects at different frequencies are predicted in figure 17. Inside the effective frequency band $(1000 \mathrm{~Hz}-6000 \mathrm{~Hz})$, from an overall point of view, the focusing effects improve with the increase of distance. At distances larger than or equal to $0.3 \mathrm{~m}$, the $\max (E e r)$ at frequencies inside the effective band are obviously larger than that at limit frequencies $(100 \mathrm{~Hz}$ and $8000 \mathrm{~Hz}$ ). Therefore the distance of the point force should be larger than $0.3 \mathrm{~m}$ for achieving acceptable focussing effects, which is almost one and a half times the longest wavelength of the considered effective frequencies.

The variations of the energy concentration zone and energy enhancement zone with the point force distance at $2000 \mathrm{~Hz}$ are illustrated in figure 18. It can be observed that as the distance increases the energy will be focused closer to the designed focal point and will be more concentrated. Consequently, the energy enhancement zone shifts to the left and shrinks.

The piezo-lens can also focus waves generated by point force away from the central axis. According to the performances of the piezo-lens for oblique plane waves and waves excited by point forces on the central axis, it has limitations for the location of the paraxial point force. The vertical distance of the point force to the left piezo-lens boundary should be at least one and a half times larger than the longest wavelength and the vertical distance of the point force to the central axis should guarantee that the incident angles of the waves are smaller than $40^{\circ}$. Even with these limitations, the point force is available in a large zone. As an example, shown in figure 19 are the focusing effects for waves excited by a point force located $1 \mathrm{~m}$ away from the piezo-lens and $0.2 \mathrm{~m}$ away from the central axis. As expected, the piezo-lens is effective in a large frequency band. Energy is concentrated away from the designed focal point and the energy concentration zone shifts to the right with the increase of frequency. Similar to the oblique plane wave cases, multiple focalization is observed at a higher frequency.

\subsection{Double piezo-lens configuration}

The piezo-lens has good focusing effects for waves generated by a point force located sufficiently far away, whereas the wave fields generated by point force are scattered, and the energy reduces with the wave travelling distance. Even though energy can be significantly enhanced after the lens, the magnitude of the energy could be still unacceptable. Thus, it is more practical to focus waves near the point source. The wave propagation in the flat GRIN lens zone is reversible, i.e. 

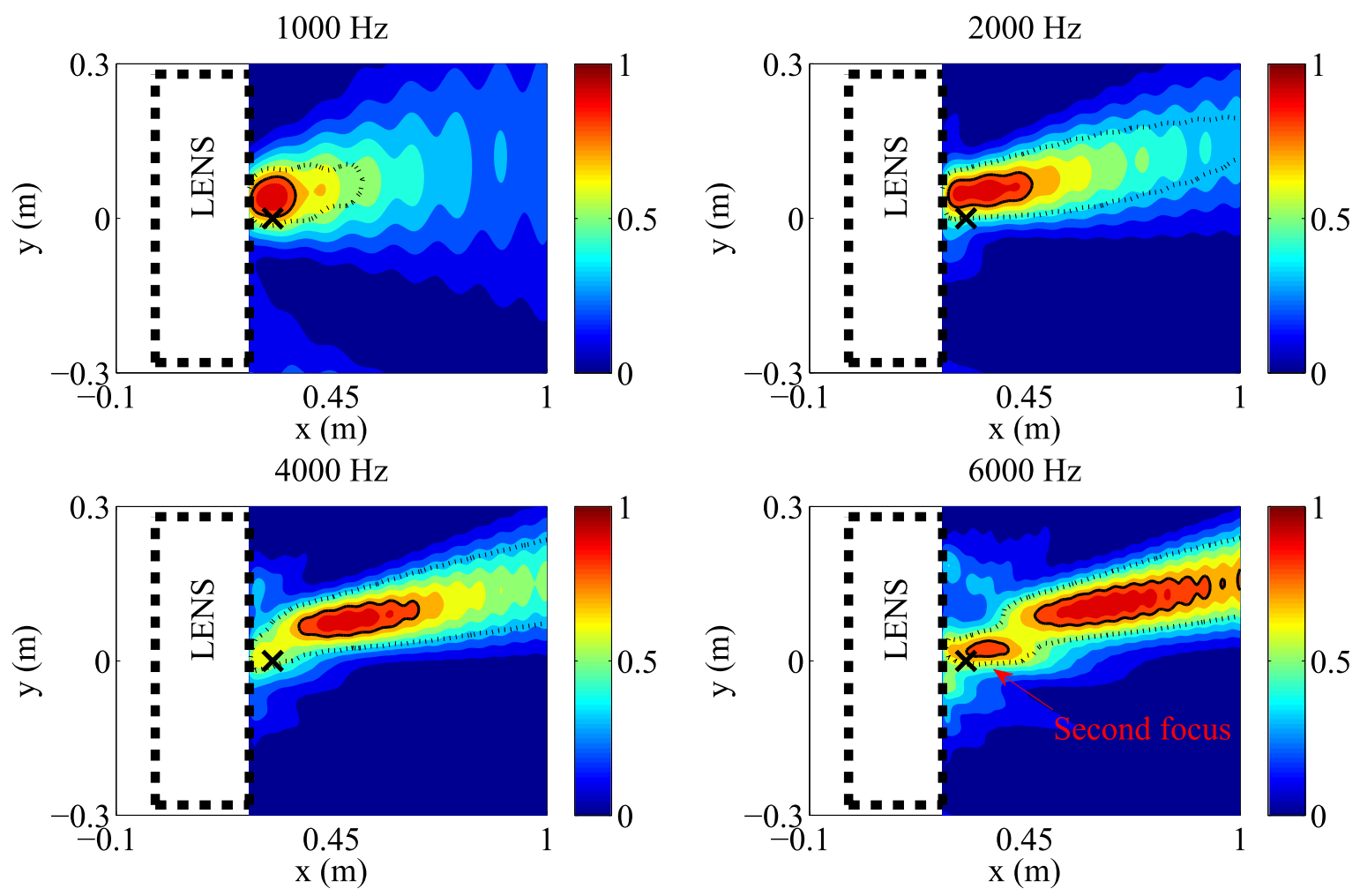

Figure 19. Normalized kinetic energy distributions at different frequencies for waves excited by paraxial point force. Black crosses indicate the designed focal points, solid and dashed lines indicate the energy concentration zones and the energy enhancement zones, respectively.
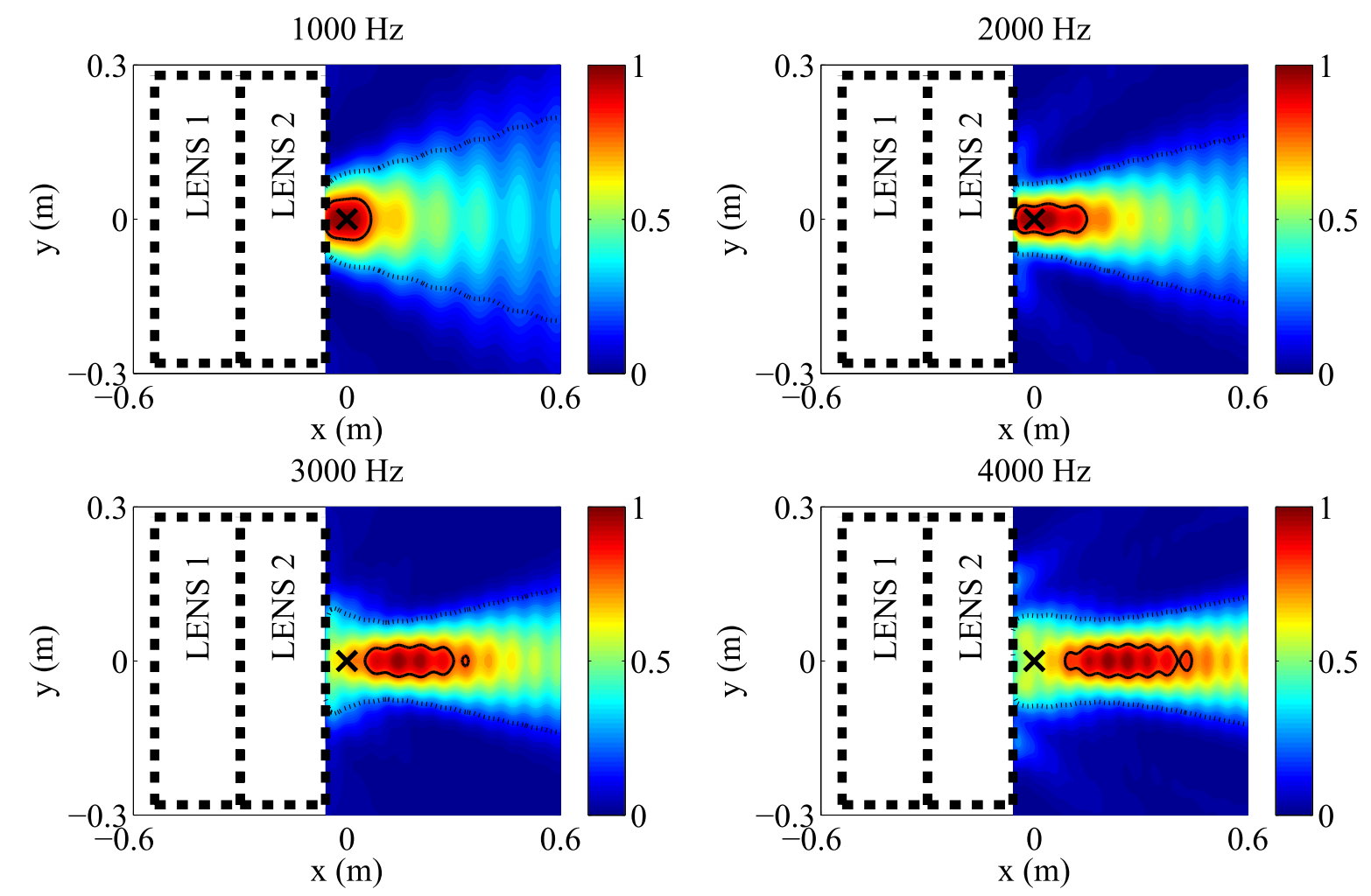

Figure 20. Normalized kinetic energy distributions after the double piezo-lens configuration for near field point force at different frequencies. The black crosses indicate the designed focal points, the solid and dashed lines indicate the energy concentration zones and the energy enhancement zones, respectively. 


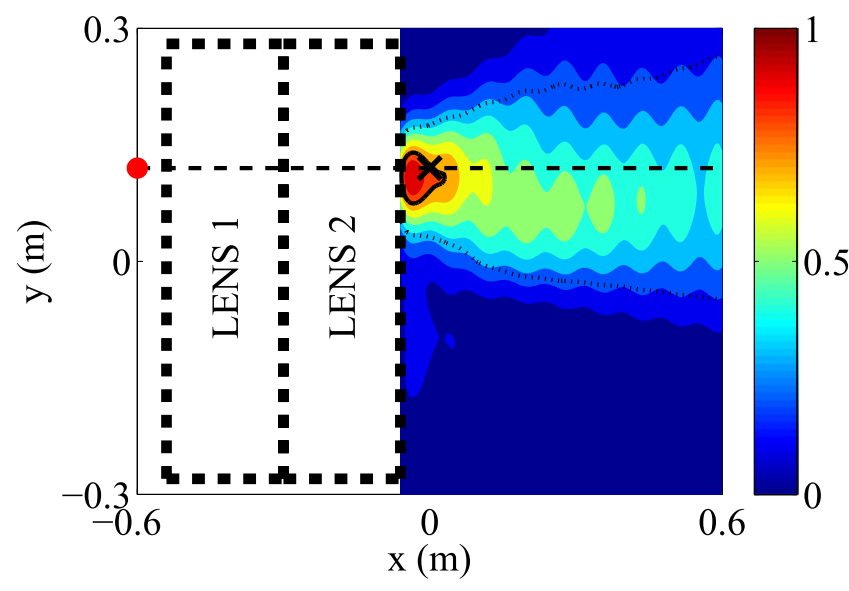

(a) $\alpha=\pi / 0.6, \beta=0.12$

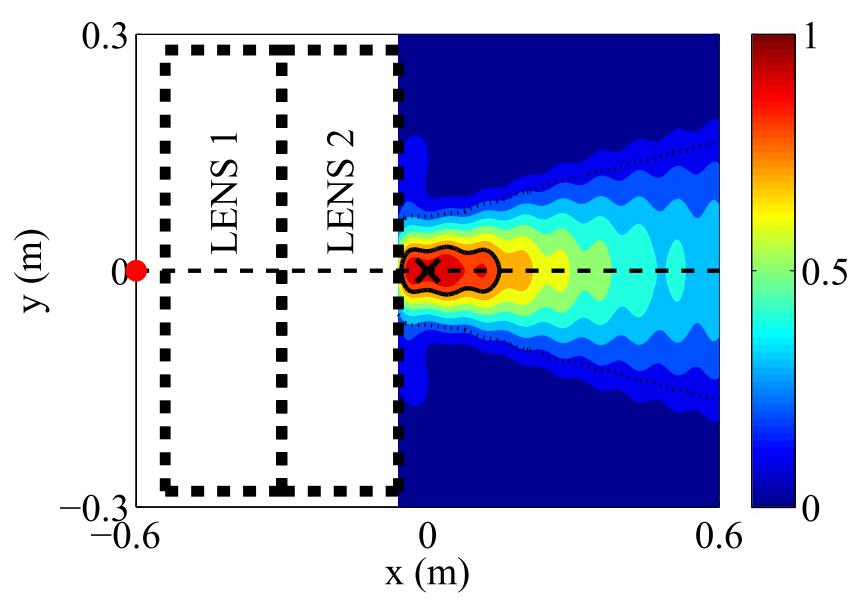

(b) $\alpha=\pi / 0.6, \beta=0$

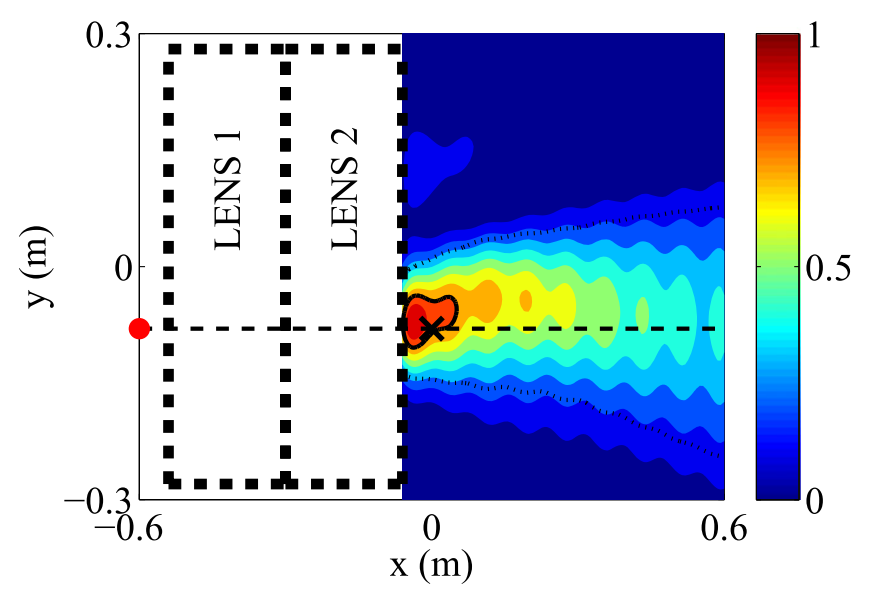

(c) $\alpha=\pi / 0.6, \beta=-0.08$

Figure 21. Normalized kinetic energy distributions after the double piezo-lens configurations at $2000 \mathrm{~Hz}$ for different focal locations.

The black crosses indicate the designed focal points, the red points indicate the locations of the point forces, the solid and dashed lines indicate the energy concentration zones and the energy enhancement zones, respectively.

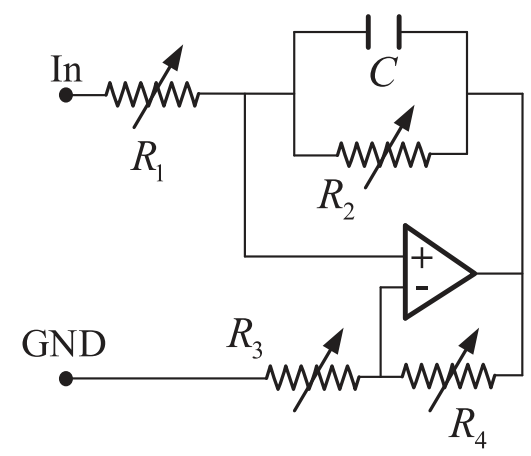

Figure A1. Real-life negative capacitance circuit used in Tateo et al's works $[29,30]$.

waves generated by a point force located at the designed focal point will be adjusted to parallel with the central axis by the lens. According to this, a double piezo-lenses configuration is proposed here.

In this configuration, an identical piezo-lens depicted in figure 1 is positioned just after the original one. The first piezo-lens is used to bend the waves generated by a point force at the designed focal point to be parallel with the central axis. The second piezo-lens is then used to focus these waves. The efficiency of this proposition is demonstrated by an example below. Set the parameters as $\alpha=\pi / 0.6$ and $\beta=0$ for each piezo-lens in the double-lens configuration. The focal point on the left side is $0.06 \mathrm{~m}$ away from the left boundary on the $y=0$ line and a point force is located at it. The focusing effects at different frequencies are demonstrated in figure 20 .

Due to the adaptive ability, the double piezo-lens configuration can focus energy at different locations, as demonstrated in figure 21. In these examples, the two piezo-lenses in a double-lens configuration are identical. Parameter $\alpha$ is set as $\alpha=\pi / 0.6$ for all the double piezo-lens configurations, but parameter $\beta$ is chosen differently as $\beta=0.12, \beta=0$ and $\beta=-0.08$ to focus waves at a distance $0.3 \mathrm{~m}$ on the $y=0.12$ line, $y=0$ line and $y=-0.08$ line, respectively. Flexural waves are excited by point forces located at the corresponding left focal points at these three cases as depicted in the figure.

\section{Conclusion and discussion}

The most remarkable conclusions obtained from the numerical results in this paper are summarized below with corresponding discussions.

- The piezo-lens is effective in a frequency band; the lower limit frequency is dominated by the characteristic length of the piezo-lens, and the upper one is limited by the length of the lattice. The effective frequency band can be extended by decreasing the length of the lattice. Inside the effective frequency band, the focal zone will shift towards 


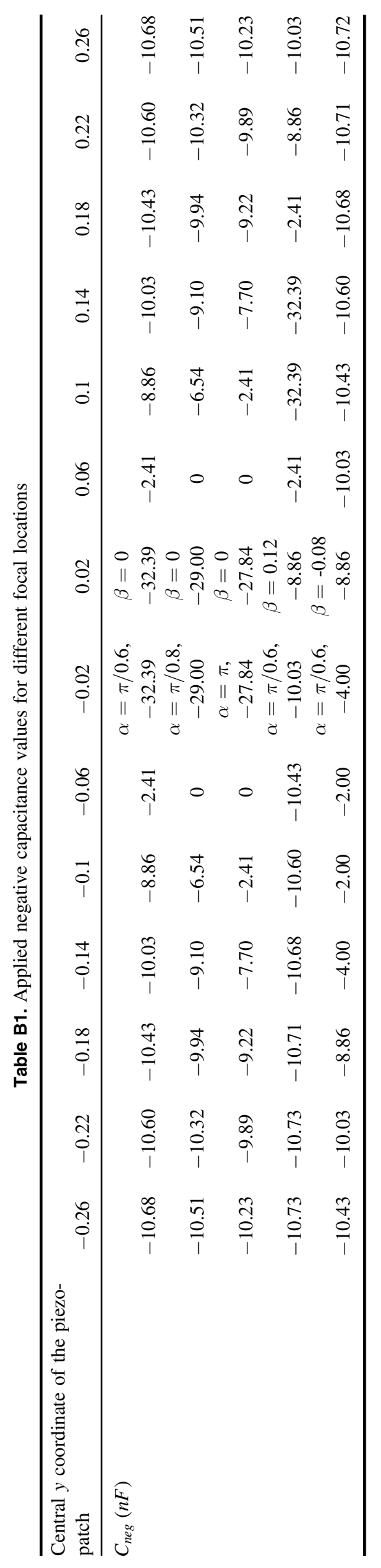


the right and enlarges as the frequency increases. But energy can almost be focused near the designed location if the wavelength is larger than four times the lattice's length.

A large effective frequency band makes the piezolens quite promising in broadband energy harvesting systems [38].

- With the same geometry configuration, the piezo-lens can focus waves at different locations by tuning the shunting negative capacitance values.

This adaptive ability distinguishes the piezo-lens from other existing GRIN lenses. With this ability, the piezo-lens can be used in SHM to simultaneously monitor a large region [39].

- The piezo-lens is effective for obliquely incident plane waves and waves excited by point forces in a broadband. The incident angle is available in a broad range and the point force can be located in a large zone.

In the existing literature, flat GRIN lenses were only verified to be effective in focusing plane waves incident from the normal direction. The effectivity of the piezolens for waves generated by different types of sources demonstrates that the piezo-lens can be applied in other cases.

- The proposed double piezo-lens configuration is effective for waves excited by point forces located in the near field in a large frequency band and can concentrate energy at different locations by adjusting the negative capacitance values.

The double piezo-lens configuration is more practical for point source cases. The characteristics summarized above make it useful in broadband energy harvesting systems and SHM.

\section{Acknowledgments}

The first author sincerely thanks a scholarship provided by the China Scholarship Council.

\section{Appendix A. Tuning the negative capacitance values in real-life applications}

In practice, the negative capacitance value can be realized by a synthetic circuit as used in Tateo et al's works [29, 30]. The layout of this circuit is described in figure A1. It contains a number of passive components, four resistors $R_{1}, R_{2}, R_{3}, R_{4}$ and a capacitor $C$, as well as an operational amplifier (OpAmp). The equivalent impedance of this circuit is :

$$
Z_{e q}(\omega)=R_{1}-\frac{R_{3}}{R_{4}} \frac{1}{j \omega C+\frac{1}{R_{2}}} .
$$

Here, $\sqrt{j}=-1$. According to Tateo et al, the formula in equation (19) can be simplified without loss of generality. First, the resistor $R_{2}$, which is necessary for the stability of the
Op-Amp at DC, is sufficiently large to be considered negligible. Second, the resistor $R_{1}$, required in practical realization of the circuit, can be slected small enough to be negligible. Therefore, the equivalent impedance in equation (19) can be simplified as:

$$
Z_{e q}(\omega)=-\frac{R_{3}}{R_{4}} \frac{1}{j \omega C}=\frac{1}{j \omega C_{n e g}} .
$$

Thus, a required negative capacitance value $C_{n e g}=-\frac{R_{4} C}{R_{3}}$ can be obtained by tuning the values of $R_{3}, R_{4}$ and $C$ in the circuit in figure A1.

\section{Appendix B. Applied negative capacitance values in simulations for different focal locations}

The negative capacitance values used in the simulations to demonstrate the adaptive ability of the piezo-lens are listed in table B1. For reference, the value set corresponding to the unstable zone is $[-11.82,-10.74] n F$.

\section{References}

[1] Yan X, Zhu R, Huang G and Yuan F G 2013 Focusing guided waves using surface bonded elastic metamaterials Appl. Phys. Lett. 103121901

[2] Jin Y, Torrent D, Pennec Y, Pan Y and Djafari-Rouhani B 2015 Simultaneous control of the S0 and A0 Lamb modes by graded phononic crystal plates J. Appl. Phys. 117244904

[3] Luo C, Johnson S, Joannopoulos J and Pendry J 2002 Allangle negative refraction without negative effective index Phys. Rev. B $652-5$

[4] Zhou X, Assouar M B and Oudich M 2014 Acoustic superfocusing by solid phononic crystals Appl. Phys. Lett. 105233506

[5] Farhat Md, Guenneau S, Enoch S, Movchan A B and Petursson G G 2010 Focussing bending waves via negative refraction in perforated thin plates Appl. Phys. Lett. 96 $18-21$

[6] Dubois M, Farhat M, Bossy E, Enoch S, Guenneau S and Sebbah P 2013 Flat lens for pulse focusing of elastic waves in thin plates Appl. Phys. Lett. 103 1-4

[7] Merchand E W 1978 Gradient Index Optics (New York: Academic)

[8] Lin Sz C S, Huang T J, Sun J H and Wu T T 2009 Gradientindex phononic crystals Phys. Rev. B 79094302

[9] Lin S C S, Tittmann B R and Huang T J 2012 Design of acoustic beam aperture modifier using gradient-index phononic crystals J. Appl. Phys. 111123510

[10] Climente A, Torrent D and Sánchez-Dehesa J 2010 Sound focusing by gradient index sonic lenses Appl. Phys. Lett. 97 $1-4$

[11] Wu T T, Chen Y T, Sun J H, Lin Sz C S and Huang T J 2011 Focusing of the lowest antisymmetric Lamb wave in a gradient-index phononic crystal plate Appl. Phys. Lett. 98 171911

[12] Chiou M J, Lin Yu C, Ono T, Esashi M, Yeh S L and Wu T T 2014 Focusing and waveguiding of Lamb waves in microfabricated piezoelectric phononic plates Ultrasonics $\mathbf{5 4}$ 1984-90 
[13] Climente A, Torrent D and Sánchez-Dehesa J 2014 Gradient index lenses for flexural waves based on thickness variations Appl. Phys. Lett. 105064101

[14] Moheimani S O R 2003 A survey of recent innovations in vibration damping and control using shunted piezoelectric transducers IEEE Trans. Control Syst. Technol. 11 482-94

[15] Hagood N W and von Flotow A 1991 Damping of structural vibrations with piezoelectric materials and passive electrical networks $J$. Sound Vib. 146 243-68

[16] Wu S Y 1998 Method for multiple mode shunt damping of structural vibration using a single PZT transducer Proc. SPIE 3327 159-68

[17] Li L, Yin S, Liu X and Li J 2015 Enhanced electromechanical coupling of piezoelectric system for multimodal vibration Mechatronics 31 205-14

[18] Park C H 2005 Vibration Control of Beams with Negative Capacitive Shunting of Interdigital Electrode Piezoceramics J. Vib. Control 11 331-46

[19] Behrens S, Fleming A J and Moheimani S O R 2003 A broadband controller for shunt piezoelectric damping of structural vibration Smart Mater. Struct. 12 18-28

[20] Casadei F, Ruzzene M, Dozio L and Cunefare K a 2009 Broadband vibration control through periodic arrays of resonant shunts: experimental investigation on plates Smart Mater. Struct. 19015002

[21] Alessandroni S, Andreaus U, Dell'Isola F and Porfiri M 2005 A passive electric controller for multimodal vibrations of thin plates Comput. Struct. 83 1236-50

[22] Thorp O, Ruzzene M and Baz a 2001 Attenuation and localization of wave propagation in rods with periodic shunted piezoelectric patches Smart Mater. Struct. 10 979-89

[23] Airoldi L and Ruzzene M 2011 Design of tunable acoustic metamaterials through periodic arrays of resonant shunted piezos New J. Phys. 13113010

[24] Chen S, Wang G, Wen J and Wen X 2013 Wave propagation and attenuation in plates with periodic arrays of shunted piezo-patches J. Sound Vib. 332 1520-32

[25] Chen Y Y, Huang G L and Sun C T 2014 Band Gap Control in an Active Elastic Metamaterial With Negative Capacitance Piezoelectric Shunting J. Vib. Acoust. 136061008

[26] Celli P and Gonella S 2015 Tunable directivity in metamaterials with reconfigurable cell symmetry Appl. Phys. Lett. 106091905

[27] Collet M, Cunefare KA K a A and Ichchou M 2008 Wave Motion Optimization in Periodically Distributed Shunted
Piezocomposite Beam Structures J. Intell. Mater. Syst. Struct. 20 787-808

[28] Collet M, Ouisse M and Ichchou M N 2012 Structural energy flow optimization through adaptive shunted piezoelectric metacomposites J. Intell. Mater. Syst. Struct. 23 1661-77

[29] Tateo F, Collet M, Ouisse M and Cunefare K 2014 Design variables for optimizing adaptive metacomposite made of shunted piezoelectric patches distribution J. Vib. Control 22 $1838-54$

[30] Tateo F, Collet M, Ouisse M, Ichchou M, Cunefare K and Abbe P 2015 Experimental characterization of a bidimensional array of negative capacitance piezo-patches for vibroacoustic control J. Intell. Mater. Syst. Struct. 26 952-64

[31] Fan Y, Collet M, Ichchou M, Li L, Bareille O and Dimitrijevic Z 2016 Energy flow prediction in built-up structures through a hybrid finite element/wave and finite element approach Mech. Syst. Signal Process. 66 137-58

[32] Livet S, Collet M, Berthillier M, Jean P and Cote J-M 2011 Structural multi-modal damping by optimizing shunted piezoelectric transducers Eur. J. Comput. Mech. 20 73-102

[33] Zhang H, Wen J, Xiao Y, Wang G and Wen X 2015 Sound transmission loss of metamaterial thin plates with periodic subwavelength arrays of shunted piezoelectric patches J. Sound Vib. 343 104-20

[34] Reddy J N 2004 Mechanics of Laminated Composite Plates and Shells: Theory and Analysis 2nd edn (New York: CRC Press)

[35] Basu U and Chopra A K 2003 Perfectly matched layers for time-harmonic elastodynamics of unbounded domains: Theory and finite-element implementation Comput. Methods Appl. Mech. Eng. 192 1337-75

[36] Chang Z, Guo D K, Feng X Q and Hu G K 2014 A facile method to realize perfectly matched layers for elastic waves Wave Motion 51 1170-8

[37] Zhao J, Bonello B, Marchal R and Boyko O 2014 Beam path and focusing of flexural Lamb waves within phononic crystal-based acoustic lenses New J. Phys. 16063031

[38] Sodano H A, Inman D J and Park G 2004 A Review of Power Harvesting from Vibration Using Piezoelectric Materials The Shock and Vibration Digest 36 197-205

[39] Baravelli E, Senesi M, Ruzzene M, DeMarchi L and Speciale N 2011 Double-channel, frequency-steered acoustic transducer with 2-D imaging capabilities IEEE Trans. Ultrason. Ferroelectr. Freq. Control 58 1430-41 